\title{
The Law of $O$-Conditionality for Fuzzy Implications Constructed from Overlap and Grouping Functions
}

\author{
Graçaliz Pereira Dimuro $^{\mathrm{a}, *}$, Benjamín Bedregal ${ }^{\mathrm{b}}, \mathrm{Javier}_{\text {Fernandez }}^{\mathrm{c}}$, Mikel Sesma-Sara ${ }^{\mathrm{c}}$, Jesús M. Pintor ${ }^{\mathrm{d}}$, Humberto \\ Bustince $^{\mathrm{c}}$ \\ ${ }^{a}$ Centro de Ciência Computacionais, Universidade Federal do Rio Grande \\ Av. Itália km 08, Campus Carreiros, 96201-900 Rio Grande, Brazil \\ ${ }^{b}$ Departamento de Informática e Matemática Aplicada, Universidade Federal do Rio Grande do Norte \\ Campus Universitário s/n, 59072-970 Natal, Brazil \\ ${ }^{c}$ Departamento de Automática y Computación and Institute of Smart Cities \\ Universidad Pública de Navarra \\ Campus Arrosadia s/n, 31006, Pamplona, Spain \\ ${ }^{d}$ Departamento de Ingeniería Mecánica, Energética y de Materiales \\ Universidad Pública de Navarra \\ Campus Arrosadia s/n, 31006, Pamplona, Spain
}

\begin{abstract}
Overlap and grouping functions are special kinds of non necessarily associative aggregation operators proposed for many applications, mainly when the associativity property is not strongly required. The classes of overlap and grouping functions are richer than the classes of t-norms and t-conorms, respectively, concerning some properties like idempotency, homogeneity, and, mainly, the self-closedness feature with respect to the convex sum and the aggregation by generalized composition of overlap/grouping functions. In previous works, we introduced some classes of fuzzy implications derived by overlap and/or grouping functions, namely, the residual implications $R_{O}$-implications, the strong implications $(G, N)$-implications and the Quantum Logic implications $Q L$-implications, for overlap functions $O$, grouping functions $G$ and fuzzy negations $N$. Such implications do not necessarily satisfy certain properties, but only weaker versions of these properties, e.g., the exchange principle. However, in general, such properties are not demanded for many applications. In this paper, we analyze the so-called law of $O$-Conditionality, $O(x, I(x, y)) \leq y$, for any fuzzy implication $I$ and overlap function $O$, and, in particular, for $R_{O}$-implications, $(G, N)$-implications, $Q L$-implications and $D$-implications derived from tuples $(O, G, N)$, the latter also introduced in this paper. We also study the conditional antecedent boundary condition for such fuzzy implications, since we prove that this property, associated to the left ordering property, is important for the analysis of the $O$-Conditionality. We show that the use of overlap functions to implement de generalized Modus Ponens, as the scheme enabled by the law of $O$-Conditionality, provides more generality than the laws of $T$-conditionality and $U$-conditionality, for t-norms $T$ and uninorms $U$, respectively.
\end{abstract}

Keywords: Overlap functions, grouping functions, fuzzy implications, $O$-conditionality, conditional antecedent boundary condition.

\section{Introduction}

Fuzzy implications [1] generalize the classical implication to fuzzy logic, by considering truth values varying in the unit interval $[0,1]$ instead of in the set $\{0,1\}$. Among several applications (see, e.g: $[2,3,4]$ ), fuzzy implications are largely applied in approximate reasoning, being used for modelling fuzzy conditionals and also in the

\footnotetext{
* Corresponding author. Phone: +555332336685

Email addresses: gracaliz@furg.br; gracaliz@gmail.com (Graçaliz Pereira Dimuro), bedregal@dimap.ufrn.br (Benjamín Bedregal), fcojavier.fernandez@unavarra.es (Javier Fernandez), mikel. sesma@unavarra.es (Mikel Sesma-Sara), txma@unavarra.es (Jesús M. Pintor), bustince@unavarra.es (Humberto Bustince)
} 
inference processes via the generalized Modus Ponens (GMP) and Modus Tollens (GMT), carried out via the Zadeh's Compositional Rule of Inference [5]. ${ }^{1}$

The GMP, which is the focus of this paper, can be implemented by a scheme enabled by the functional inequality called the Law of $T$-Conditionality, which, for a t-norm $T$ and a fuzzy implication $I$, is stated by:

(TC) $\forall x, y \in[0,1]: T(x, I(x, y)) \leq y$.

In fact, (TC) means $x *_{T}(x \rightarrow y) \leq y$, which generalizes the Modus Ponens $x \wedge_{T}(x \rightarrow y) \vdash y$ to the fuzzy context $[7,8]$.

In the literature, the studies on the $T$-Conditionality have been done just for the three main families of fuzzy implications, namely, $R$-, $(S, N)$ - and $Q L$-implications. ${ }^{2} R$-implications are generalizations to $[0,1]$ of Boolean implications defined by the identity given, for a universe set $X$, by

$$
A^{\prime} \cup B=(A-B)^{\prime}=\bigcup\{C \subseteq X \mid(A \cap C) \subseteq B\},
$$

where $A, B \subseteq X$, and the intersection is generalized by a t-norm. This class of implications is related to a residuation concept from the intuitionistic logic. On the other hand, $(S, N)$-implications are generalizations to $[0,1]$ of the Boolean material implication defined as

$$
p \rightarrow q \equiv \neg p \vee q,
$$

where $\vee$ and $\neg$ are replaced, respectively, by a t-conorm $S$ and a fuzzy negation $N$. Finally, $Q L$-implications are generalizations to $[0,1]$ of the implication

$$
p \rightarrow q \equiv \neg p \vee(p \wedge q)
$$

defined in quantum logic, where $\wedge$ is replaced by a t-norm, $\vee$ by a t-conorm and $\neg$ by a fuzzy negation. However, $D$-implications, which are generalization to $[0,1]$ of the Dishkant implication

$$
p \rightarrow q \equiv q \vee(\neg p \wedge \neg q)
$$

of orthomodular lattices, is also an important family of fuzzy implications attracting attention in the literature [1,3].

Observe that t-norms and t-conorms require the associativity and commutativity properties, which, in their turn, allow any $R$-implication and $(S, N)$-implication $I$ to satisfy the exchange principle $I(x, I(y, z))=I(y, I(x, z))$. $(S, N)$-implications also satisfy the left neutrality principle $I(1, y)=y$, as well as $Q L$-implications [1].

However, in the literature, it was shown that the associativity property of the conjuctive operator is not demanded for many applications, e.g., in pairwise comparisons, image processing, fuzzy rule-based classification systems, entropy and mathematical morphology $[4,10,11,12,13,14]$. Similarly, the exchange principle is not required for fuzzy implication functions in several applications, e.g., in decision making [15, 16, 17], consensus measures [15, 16], multicriteria decision problem by similarity measures [18]. See the discussions in $[4,10,19,20,21]$ and, for a summary of related works discussing the subject, see [22, 23, 24, 25, 26].

As an example, in fuzzy modeling of pairwise comparisons [27, 28], in the context of decision making based on fuzzy preference relations [29, 30, 31, 32], in general, an indifference relation is defined using a weak preference relation given by a t-norm combination, which is a measures of the the (weak) preference of one alternative over the other and vice versa $[33,34]$. However, a pairwise comparison refers to only two alternatives (and the result of the comparison is not an alternative itself), and so the associative property is not a strong requirement, and then, one need not use t-norms as the combination operator [35]. Some analogous arguments may be given for classification problems, as in image processing, entropy and mathematical morphology.

Another interesting example is when the conjunctive operator is replaced by an implication function, where the exchange principle is not required, since just two arguments are considered, and the resulting decision is not an

\footnotetext{
${ }^{1}$ For methods to solve GMP, also called by fuzzy Modus Ponens, see [6].

${ }^{2}$ We remark that those studies were done under some restricted conditions, e.g., the continuity of the underlying operators is, in general, assumed $[1,9]$.
} 
argument itself, as the work by Beliakov et al. [15, 16], who applied fuzzy implications and aggregation functions to construct more flexible consensus measures, avoiding the symmetry property of similarity and distance functions that could, in some circumstances, be too restrictive when considering weighted consensus models.

The discussion above justifies the use of other kinds of aggregation functions that are able to model conjunctions, disjunctions and implications in some way in a more general context (see, e.g., the works by Ouyang [36], Pradera et al. [37] and Pinheiro et al. [38]).

In this direction, Bustince et al. [12,35] introduced overlap and grouping functions that are particular cases of continuous aggregation operators given by (not necessarily associative) increasing commutative functions, satisfying appropriate boundary conditions. Overlap and grouping functions were extensively studied in the literature. See, e.g., $[19,20,23,25,26,39,40,41]$.

Then, in the sequence, the concept of fuzzy implications derived from overlap and grouping functions were introduced. In [21, 42], based on residual implicators of general conjunctions [43, 44], Dimuro et al. introduced $R_{O}$-implications, the residual implications derived from overlap functions $O$, preserving the residuation property. The concept of $(G, N)$-implication, for a grouping function $G$ and a fuzzy negation $N$, was introduced by Dimuro et al. in [4], and $Q L$-implications derived from tuples $(O, G, N)$ were introduced in [10], where it was showed an application to the generation of fuzzy subsethood and entropy measures. Such implications do not necessarily satisfy certain properties, e.g., the exchange principle (for $R_{O}$-implications), or the exchange and the left neutrality principles (for $(G, N)$-implications) or just the left neutrality property (for $Q L$-implications), but only weaker versions of these properties. However, in general, such properties are not demanded for many applications [4, 21], and, in particular, for the subject of this paper.

There are many advantages of overlap/grouping functions over t-norms/t-conorms. First notice that the classes of overlap and grouping functions are richer than the classes of t-norms and t-conorms, respectively, concerning some properties, like idempotency, homogeneity, and, mainly, the self-closedness feature with respect to the convex sum and the aggregation by generalized composition of overlap/grouping functions, as discussed in [4, 21].

Now observe that there are some applications where the concept of overlap/grouping functions are essential, e.g., in classification and image processing [12,45]. Moreover, in several applications, overlap/grouping functions and implication functions derived by them can provide more flexibility and generality, whenever associativity and the exchange principle are not required, e.g., in fuzzy modeling of pairwise comparison [35], decision making [46, 47], fuzzy subsethood measures [10,48], entropy measures [10]. Additionally, in certain applications overlap functions led to better results than t-norms, e.g., in fuzzy rule based classification problems [11, 14, 49, 50, 51, 52, 53]).

As an example, the classification problems that arise, for instance, in image processing, naturally encompass the overlapping problem. Overlap functions measure the degree of overlap between the two functions that represent the object and background, which can be interpreted as the representation of the lack of knowledge of an expert in determining if a certain pixel belongs to the object or to the background $[12,45]$.

Another example is the fuzzy modeling of pairwise comparisons in decision making based on fuzzy preference relations, where Bustince et al. introduced in [35] some construction methods for the concept of indifference (in the sense of [33, 34]) defined in terms of overlap functions. Also, grouping functions are used to measure the amount of evidence in favor of either of the two alternatives. Thus, its negation provides a measure of incomparability.

Considering implication functions derived from overlap and grouping functions, they have been used, for example, for defining subsethood and entropy measures [10], and extensions to the interval-valued context were also provided by Cao et al. [24].

Concerning the advantages and flexibility provided by overlap functions as conjunctive operators, in the present paper, we introduce a generalization of (TC), substituting the t-norms and fuzzy implications by, respectively, overlap functions and fuzzy implications derived from overlap and grouping functions, in order to provide more generality of the implementation of GMP (see Example 4.1).

Then, the objectives of this paper are: (i) to introduce the concept of $D$-operations and implications derived from tuples $(O, G, N)$, studying some properties that are useful for the purpose of this paper; (ii) to analyze the law of $O$-Conditionality, defined by

$$
O(x, I(x, y)) \leq y,
$$

for any fuzzy implication $I$ and overlap function $O$; (iii) to study the conditional antecedent boundary condition for $R_{O^{-}},(G, N)$-, $Q L$ - and $D$-implications derived from tuples $(O, G, N)$, since we prove that this property, associated 
to the left ordering property ${ }^{3}$, is important for the analysis of the $O$-Conditionality (see Section 4 ); (iv) to analyze the law of $O$-Conditionality for $R_{O^{-}},(G, N)$-, $Q L$ - and $D$-implications derived from tuples $(O, G, N)$.

The paper is organized as follows. Section 2 presents basic concepts that are necessary to develop the paper, including the concepts related to $R_{O^{-}},(G, N)$-, $Q L$-operations and implications derived from tuples $(O, G, N)$, in order to make this paper self-contained. In Section 3, we introduce the concept of $D$-operations and implications derived from tuples $(O, G, N)$, studying some properties. The $O$-conditionality for fuzzy implications in general is analysed in Section 4. The study of the conditional antecedent boundary condition for $R_{O^{-}},(G, N)$-, $Q L$-implications and $D$-implications derived from tuples $(O, G, N)$ is presented in Section 5. The $O$-conditionality for $R_{O^{-}},(G, N)$-, $Q L$ - and $D$-implications derived from tuples $(O, G, N)$ is analysed in Section 6. Section 7 is the Conclusion, with our final remarks and outlining future work.

\section{Preliminary Concepts}

In this section, we present the basic concepts that are necessary to develop the paper, including the $R_{O^{-}},(G, N)$, $Q L$-operations and implications derived from tuples $(O, G, N)$.

2.1. Aggregation functions: $t$-norms, $t$-conorms, overlap and grouping functions

Definition 2.1. A function $N:[0,1] \rightarrow[0,1]$ is said to be a fuzzy negation if the following conditions hold:

(N1) $N$ satisfies the Boundary Conditions: $N(0)=1$ and $N(1)=0$;

(N2) $N$ is decreasing: if $x \leq y$ then $N(y) \leq N(x)$.

A fuzzy negation is called strict if, in addition,

(N3) $N$ is strictly decreasing;

(N4) $N$ is continuous.

A fuzzy negation is called strong if it is involutive, that is,

(N5) $\forall x \in[0,1]: N(N(x))=x$.

A fuzzy negation is called frontier if it satisfies the property:

(N6) $N(x) \in\{0,1\}$ if and only if $x=0$ or $x=1$.

A fuzzy negation is called non-filling if it satisfies the property [1]:

(N7) $N(x)=1$ if and only if $x=0$.

Examples of fuzzy negations are the standard fuzzy negation or Zadeh negation $N_{Z}:[0,1] \rightarrow[0,1]$, defined by $N_{Z}(x)=1-x$, the least fuzzy negation $N_{\perp}:[0,1] \rightarrow[0,1]$

$$
N_{\perp}(x)= \begin{cases}1 & \text { if } x=0 \\ 0 & \text { if } x \in] 0,1] .\end{cases}
$$

and the greatest fuzzy negation $N_{\top}:[0,1] \rightarrow[0,1]$

$$
N_{\top}(x)= \begin{cases}0 & \text { if } x=1, \\ 1 & \text { if } x \in[0,1[.\end{cases}
$$

Definition 2.2. A function $A:[0,1]^{n} \rightarrow[0,1]$ is said to be an n-ary aggregation function if:

\footnotetext{
${ }^{3}$ The left ordering property for $R_{O^{-}},(G, N)$ - and $Q L$-implications derived from tuples $(O, G, N)$ was already studied in our previous works [4, $10,21]$.
} 
(A1) $A$ is increasing ${ }^{4}$ in each argument: for each $i \in\{1, \ldots, n\}$, if $x_{i} \leq y$, then

$$
A\left(x_{1}, \ldots, x_{n}\right) \leq A\left(x_{1}, \ldots, x_{i-1}, y, x_{i+1}, \ldots, x_{n}\right)
$$

(A2) A satisfies the boundary conditions: $A(0, \ldots, 0)=0$ and $A(1, \ldots, 1)=1$.

Definition 2.3. A bivariate aggregation operator $T:[0,1]^{2} \rightarrow[0,1]$ is a $t$-norm if, for all $x, y, z \in[0,1]$, it satisfies the following properties:

(T1) Commutativity: $T(x, y)=T(y, x)$;

(T2) Associativity: $T(x, T(y, z))=T(T(x, y), z)$;

(T3) Boundary condition: $T(x, 1)=x$.

An element $x \in] 0,1]$ is said to be a non-trivial zero divisor of $T$ if there exists $y \in] 0,1]$ such that $T(x, y)=0$. A t-norm is positive if and only if it has no non-trivial zero divisors, that is, if $T(x, y)=0$ then either $x=0$ or $y=0$. A typical example of continuous and positive t-norm is the minimum t-norm $T_{M}:[0,1]^{2} \rightarrow[0,1]$, defined by $T_{M}(x, y)=\min \{x, y\}$.

Definition 2.4. A bivariate aggregation operator $S:[0,1]^{2} \rightarrow[0,1]$ is a $t$-conorm if, for all $x, y, z \in[0,1]$, it satisfies the following properties:

(S1) Commutativity: $S(x, y)=S(y, x)$;

(S2) Associativity: $S(x, S(y, z))=S(S(x, y), z)$;

(S3) Boundary condition: $S(x, 0)=x$.

An element $x \in[0,1[$ is said to be a non-trivial one divisor of $S$ if there exists $y \in[0,1[$ such that $S(x, y)=1$. A t-conorm is positive if and only if it has no non-trivial one divisors, that is, if $S(x, y)=1$ then either $x=1$ or $y=1$. A typical example of continuous and positive t-conorm is the maximum $S_{M}(x, y):[0,1]^{2} \rightarrow[0,1]$, defined by $S_{M}(x, y)=\max \{x, y\}$.

In this paper, the aggregation operators about which we are mainly concerned are overlap and grouping functions.

Definition 2.5. A bivariate function $O:[0,1]^{2} \rightarrow[0,1]$ is said to be an overlap function if it satisfies the conditions:

(O1) O is commutative;

(O2) $O(x, y)=0$ if and only if $x y=0$;

(O3) $O(x, y)=1$ if and only if $x y=1$;

(O4) $O$ is increasing;

(O5) O is continuous.

An overlap function $O$ is associative if and only if $O$ is a continuous and positive t-norm [12].

Definition 2.6. Let $O:[0,1]^{2} \rightarrow[0,1]$ be an overlap function:

(O6) O satisfies the property of 1-section deflation if and only if

$$
\forall x \in[0,1]: O(x, 1) \leq x
$$

\footnotetext{
${ }^{4}$ In this paper, an increasing (decreasing) function does not need to be strictly increasing (decreasing).
} 


$$
\forall x \in[0,1]: O(x, 1) \geq x .
$$

An overlap function $O$ satisfies (O6) and (O7) if and only if $O$ has 1 as neutral element. Whenever an overlap function has a neutral element, then, by $(\mathbf{O 3})$, this element is necessarily equal to 1 . The sub-class of overlap functions having 1 as neutral element is denoted by $\mathcal{O}_{x}$, and the sub-class of overlap functions satisfying the property (O6), but not necessarily having 1 as neutral element, is denoted by $\mathcal{O}_{\leq x}$.

Example 2.1. Examples of overlap functions that are used in this paper are [19, 20, 21, 39]:

$$
\begin{aligned}
O_{m M}(x, y) & =\min \{x, y\} \max \left\{x^{2}, y^{2}\right\} \\
O_{m M}^{V}(x, y) & = \begin{cases}\frac{1+O_{m M}(2 x-1,2 y-1)}{2} & \text { if } x, y \in] 0.5,1], \\
\min \{x, y\} & \text { otherwise; }\end{cases} \\
O_{p}(x, y) & =(x y)^{p}, p \geq 1 ; \\
T_{M}(x, y) & =\min \{x, y\} \text { (the Minimum t-norm); } \\
O_{m \frac{1}{2}}(x, y) & =\min \{\sqrt{x}, \sqrt{y}\} .
\end{aligned}
$$

Lemma 2.1. Let $O:[0,1]^{2} \rightarrow[0,1]$ be an overlap function. Then, for all $x, y \in[0,1]$, it holds that: then,

(i) If $O$ satisfies (O6) then $O(x, y) \leq x$;

(ii) $O$ satisfies (O6) if and only if $O \leq \min$.

PROOF. It follows that:

(i) If $O$ satisfies (O6) then, since $O$ is increasing, for all $x, y \in[0,1]$, it holds that $O(x, y) \leq O(x, 1) \leq x$.

(ii) $(\Rightarrow)$ By (i), if $O$ satisfies (O6) then, for all $x, y \in[0,1]$, it holds that $O(x, y) \leq x$. From the commutativity of $O$, we also have that $O(x, y)=O(y, x) \leq y$. Thus, one has that $O(x, y) \leq \min \{x, y\}$. $(\Leftarrow)$ It is immediate.

Definition 2.7. A bivariate function $G:[0,1]^{2} \rightarrow[0,1]$ is said to be a grouping function if it satisfies the following conditions:

(G1) $G$ is commutative;

(G2) $G(x, y)=0$ if and only if $x=y=0$;

(G3) $G(x, y)=1$ if and only if $x=1$ or $y=1$;

(G4) $G$ is increasing;

(G5) $G$ is continuous.

Definition 2.8. Let $G:[0,1]^{2} \rightarrow[0,1]$ be a grouping function.

(G6) $G$ satisfies the property of 0 -section inflation if and only if

$$
\forall x \in[0,1]: G(0, y) \geq y
$$

(G7) G satisfies the property of 0-section deflation if and only if

$$
\forall x \in[0,1]: G(0, y) \leq y .
$$


A grouping function $G$ satisfies (G6) and (G7) if and only if $G$ has 0 as neutral element. Observe that whenever a grouping function has a neutral element, then, by (G2), this element is necessarily equal to 0 . A grouping function $G$ is associative if and only if $G$ is a continuous and positive t-conorm.

Example 2.2. Examples of grouping functions that are used in this paper are [4, 19, 40]:

$$
\begin{aligned}
G_{m M}(x, y) & =1-\min \{1-x, 1-y\} \max \left\{(1-x)^{2},(1-y)^{2}\right\} \\
G_{m M}^{V}(x, y) & = \begin{cases}\frac{G_{m M}(2 x, 2 y)}{2} & \text { if } x, y \in[0,0.5[ \\
\max \{x, y\} & \text { otherwise }\end{cases} \\
G_{M p}(x, y) & =\max \left\{x^{p}, y^{p}\right\}, p>0 \\
G_{2}(x, y) & =1-(1-x)^{2}(1-y)^{2} \\
G_{D B}(x, y) & = \begin{cases}\frac{x+y-2 x y}{2-(x+y)} & \text { if } x+y \neq 2, \\
0 & \text { if } x+y=2 .\end{cases}
\end{aligned}
$$

A function $\varphi:[0,1] \rightarrow[0,1]$ is said to be an automorphism if $\varphi$ is bijective and increasing. Given a function $f:[0,1]^{n} \rightarrow[0,1]$ and an automorphism $\varphi$, the action of $\varphi$ on $f$ is the function $f^{\varphi}:[0,1]^{n} \rightarrow[0,1]$ defined by

$$
f^{\varphi}\left(x_{1}, \ldots, x_{n}\right)=\varphi^{-1}\left(f\left(\varphi\left(x_{1}\right), \ldots, \varphi\left(x_{n}\right)\right)\right) .
$$

$f^{\varphi}$ is said the conjugate of $f$.

2.2. Fuzzy implications: $R_{O^{-}},(G, N)$-, $Q$ L-operations and implications derived from tuples $(O, G, N)$

Definition 2.9. A function $I:[0,1]^{2} \rightarrow[0,1]$ is a fuzzy implication if, for each $x, y, z \in[0,1]$, it holds that:

(I1) First place antitonicity: if $x \leq y$ then $I(y, z) \leq I(x, z)$;

(I2) Second place isotonicity: if $y \leq z$ then $I(x, y) \leq I(x, z)$;

(I3) Boundary condition 1: $I(0,0)=1$;

(I4) Boundary condition 2: $I(1,1)=1$;

(I5) Boundary condition 3: $I(1,0)=0$.

There exist several properties that may be required for fuzzy implications [1, 54]. In the following, we present some properties that are used in this paper.

Definition 2.10. A fuzzy implication $I:[0,1]^{2} \rightarrow[0,1]$ satisfies:

(LBC) The left boundary condition if and only if

$$
\forall y \in[0,1]: I(0, y)=1
$$

(OP) The ordering property if and only if

$$
\forall x, y \in[0,1]: I(x, y)=1 \Leftrightarrow x \leq y ;
$$

(LOP) The left ordering property if and only if

$$
\forall x, y \in[0,1]: x \leq y \Rightarrow I(x, y)=1 ;
$$

(EP1) The exchange principle for 1 if and only if

$$
\forall x, y, z \in[0,1]: I(x, I(y, z))=1 \Rightarrow I(y, I(x, z))=1 ;
$$


(IP) The identity principle if and only if

$$
\forall x \in[0,1], I(x, x)=1 ;
$$

(PEP) The pseudo-exchange principle if and only if

$$
\forall x, y, z \in[0,1]: I(x, z) \geq y \Leftrightarrow I(y, z) \geq x ;
$$

(CAB) The conditional antecedent boundary condition if only if

$$
\forall x, y \in[0,1]: x>y \Rightarrow I(x, y) \leq y
$$

(SBC) The strong boundary conditions if and only if:

(a) $\forall x, y \in[0,1], I(x, y)=0 \Rightarrow x=1 \wedge y=0$;

(b) $\forall x, y \in[0,1], I(x, y)=1 \Rightarrow x=0 \vee y=1$;

(SBC-0) The strong boundary condition for 0 if and only if

$$
\forall x \in[0,1]: x \neq 0 \Rightarrow I(x, 0)=0 ;
$$

(NP) The left neutrality property if and only if

$$
\forall y \in[0,1], I(1, y)=y
$$

(CP) The contrapositivity property for a negation $N$ if and only if,

$$
\forall x, y \in[0,1]: I(x, y)=I(N(y), N(x)) ;
$$

(LCP) The left contrapositivity property for a negation $N$ if and only if

$$
\forall x, y \in[0,1]: I(N(x), y)=I(N(y), x) .
$$

Definition 2.11. The natural fuzzy negation of a fuzzy implication $I:[0,1]^{2} \rightarrow[0,1]$ is defined as the function $N_{I}:[0,1] \rightarrow[0,1]$, such that $N_{I}(x)=I(x, 0)$.

A fuzzy implication $I$ satisfies (SBC-0) if and only if $N_{I}=N_{\perp}$ (Equation (1)).

In the following, we summarize the concepts related to fuzzy implications derived from overlap and grouping functions introduced in our previous works [4, 10, 21, 42].

\subsection{1. $R_{O}$-Implications}

In [21], Dimuro and Bedregal studied the class of fuzzy implication functions called $R_{O}$-implication functions, where $O$ denotes overlap functions. $R_{O}$-implication functions is a sub-class of residual implications derived from fuzzy conjunctions [44, Theorem 2]. In a more general context, $R_{O}$-implication functions is a sub-class of the $R$ implication like operator $I_{A}$ constructed by means of an arbitrary binary operator $A$, introduced by Ouyang [36], who, however, has not analysed the specific case when $A$ is an overlap function.

Let $O:[0,1]^{2} \rightarrow[0,1]$ be an overlap function and define the function $I_{O}:[0,1]^{2} \rightarrow[0,1]$ by

$$
I_{O}(x, y)=\max \{z \in[0,1] \mid O(x, z) \leq y\} .
$$

From [44, Theorem 1, Theorem 2], it is immediate that:

Proposition 2.1. [21, Corollary 3.1] Let $I_{O}:[0,1]^{2} \rightarrow[0,1]$ be defined as in Equation (13). Then $I_{O}$ is a fuzzy implication. $O$ and $I_{O}$ form an adjoint pair, that is, they satisfy the residuation property:

$$
\forall x, y, u \in[0,1]: O(x, u) \leq y \Leftrightarrow I_{O}(x, y) \geq u .
$$


The fuzzy implication $I_{O}$, defined by Equation (13), is called the residual implication derived from the overlap function $O$, denoted by $R_{O}$-implication. For an $R_{O}$-implication $I_{O}$, the overlap function $O$ is said to be the generator of $I_{O} . I_{O}$ is also called as "the residuum of $\mathrm{O}$ ".

Example 2.3. Examples of $R_{O}$-implications are [1, 21]:

$$
\begin{aligned}
I_{O_{m M}^{V}}(x, y) & = \begin{cases}\min \left\{1, \max \left\{\frac{\sqrt{2 y-1}}{2 \sqrt{2 x-1}}, \frac{2 y-1}{2(2 x-1)^{2}}\right\}+\frac{1}{2}\right\} & \text { if } x \in] 0.5,1] \text { and } y \in[0.5,1] \\
y & \text { if } y \in[0,0.5[\text { and } x>y, \\
1 & \text { if } x \in[0,0.5] \text { and } x \leq y\end{cases} \\
I_{G D}(x, y) & = \begin{cases}1 & \text { if } x \leq y \\
y & \text { if } x>y\end{cases} \\
I_{O_{m \frac{1}{2}}}(x, y) & = \begin{cases}1 & \text { if } \sqrt{x} \leq y \\
y^{2} & \text { if } \sqrt{x}>y\end{cases}
\end{aligned}
$$

which are generated, respectively, by the overlap functions $O_{m M}^{V}$ (Equation (4)), $T_{M}$ (the positive and continuous Minimum t-norm given in Equation (6)) and $O_{m \frac{1}{2}}($ Equation (7)).

In $[21,42]$, several properties of $I_{O}$-implications were studied. Some of them are used in this paper, namely:

Proposition 2.2. [21, Proposition 3.1] Let $O:[0,1]^{2} \rightarrow[0,1]$ be an overlap function. Then it holds that:

(i) $I_{O}$ satisfies $(\boldsymbol{O P})$ if and only if $O$ satisfies $(\mathbf{O 6})$ and $(\mathbf{O} 7)$;

(ii) $I_{O}$ satisfies $(\mathbf{S B C}-\mathbf{0})$;

(iii) if $I_{O}$ satisfies $(\boldsymbol{O P})$ then $I_{O}$ satisfies $(\boldsymbol{E P 1})$;

(iv) $I_{O}$ satisfies $(\boldsymbol{I P})$ if and only if $O$ satisfies $(\mathbf{O 6})$;

(v) if $I_{O}$ satisfies $(\boldsymbol{O P})$ then $I_{O}$ satisfies $(\boldsymbol{P E P})$;

As a consequence, an $R_{O}$-implication satisfies the property (OP) if and only if $O$ has 1 as a neutral element [21]. In [21], we presented two characterizations of $R_{O}$-implications, for $O \in \mathcal{O}_{x}$ and $O \in \mathcal{O}_{\leq x}$ :

Theorem 2.1. [21, Theorem 4.6,Theorem 4.7] Let $I:[0,1]^{2} \rightarrow[0,1]$ be a right-continuous fuzzy implication. Then:

$\mathcal{O}_{x}$-Characterization of $R_{O}$-implications: I satisfies the properties $(\boldsymbol{O P}),(\mathbf{S B C}-\mathbf{0})$ and $(\mathbf{E P 1})$ if and only if $I$ is an $R_{O}$-implication derived from an overlap function $O \in \mathcal{O}_{x}$, that is, $I=I_{O}$, with $O \in \mathcal{O}_{x}$;

$\mathcal{O}_{\leq x}$-Characterization of $R_{O}$-implications: I satisfies the properties $(\boldsymbol{S B C}-\mathbf{0}),(\boldsymbol{I P}),(\boldsymbol{P E P})$ and $\forall y \in[0,1)$ : $I(1, y)<1$ if and only if $I$ is an $R_{O}$-implication derived from an overlap function $O \in \mathcal{O}_{\leq x}$, that is, $I=I_{O}$, with $O \in \mathcal{O}_{\leq x}$.

Proposition 2.3. Let $O_{1}, O_{2}:[0,1]^{2} \rightarrow[0,1]$ be overlap functions. $O_{1} \leq O_{2}$ if and only if $I_{O_{2}} \leq I_{O_{1}}$.

PROOF. It follows that:

$$
\begin{aligned}
O_{1} & \leq O_{2} \\
& \Leftrightarrow \quad \forall x, y, z \in[0,1]: O_{2}(x, z) \leq y \rightarrow O_{1}(x, z) \leq y \\
& \Leftrightarrow \quad \forall x, y \in[0,1]:\left\{z \in[0,1] \mid O_{2}(x, z) \leq y\right\} \subseteq\left\{z \in[0,1] \mid O_{1}(x, z) \leq y\right\} \\
& \Leftrightarrow \quad I_{O_{2}} \leq I_{O_{1}} .
\end{aligned}
$$

It is immediate that:

Corollary 2.1. Let $O_{1}, O_{2}:[0,1]^{2} \rightarrow[0,1]$ be overlap functions. $I_{O_{1}}=I_{O_{2}}$ if and only if $O_{1}=O_{2}$. 


\subsection{2. ( $G, N)$-Implications}

The class of fuzzy implications called $(G, N)$-implications, where $G$ and $N$ denote, respectively, grouping functions and fuzzy negations, were introduced by Dimuro et al. in [4]. This class of fuzzy implications is a particular subclass of $(A, N)$-functions derived from aggregation functions $A$ and fuzzy negations $N$ introduced subsequently by Pradera et al. [37, Definition 31].

Let $G:[0,1]^{2} \rightarrow[0,1]$ be a grouping function and $N:[0,1] \rightarrow[0,1]$ be a fuzzy negation, and define the function $I_{G, N}:[0,1]^{2} \rightarrow[0,1]$, by

$$
I_{G, N}(x, y)=G(N(x), y),
$$

for all $x, y \in[0,1]$.

Proposition 2.4. [4, Proposition 3.1] [37, Theorem 33] The function $I_{G, N}:[0,1]^{2} \rightarrow[0,1]$ is a fuzzy implication, called $(G, N)$-implication.

For a $(G, N)$-implication $I_{G, N}$, the grouping function $G$ and the fuzzy negation $N$ are said to be the generators of $I_{G, N}$.

Example 2.4. Some examples of $(G, N)$-implication that are used in this paper are:

(i) The grouping function $G_{m M}^{V}$ (Equation (9)) and the fuzzy negation

$$
N_{2}(x)= \begin{cases}0 & \text { if } x=1, \\ 1-\frac{x}{2} & \text { otherwise }\end{cases}
$$

generate the following $(G, N)$-implication [4]:

$$
I_{G_{m M}^{V}, N_{2}}(x, y)= \begin{cases}y & \text { if } x=1, y \in[0,0.5[, \\ \max \left\{1-\frac{x}{2}, y\right\} & \text { otherwise. }\end{cases}
$$

(ii) The grouping function $G_{M p}$ (Equation (10)), for $p=2$, and the fuzzy negation $N_{3}(x)=\sqrt{1-x}$ generate the following $(G, N)$-implication [4]:

$$
I_{G_{M p}, N_{3}}(x, y)=\max \left\{1-x, y^{2}\right\} .
$$

(iii) The grouping function $G_{M p}$ (Equation (10)) and the least fuzzy negation $N_{\perp}$ (Equation(1)) generate the following $(G, N)$-implication:

$$
I_{G_{M p}, N_{\perp}}(x, y)= \begin{cases}1 & \text { if } x=0 \\ y^{p} & \text { otherwise. }\end{cases}
$$

(iv) The grouping function $G_{2}$ (Equation (11)) and the least fuzzy negation $N_{\perp}$ (Equation(1)) generate the following $(G, N)$-implication:

$$
I_{G_{2}, N_{\perp}}(x, y)= \begin{cases}1 & \text { if } x=0 \\ 1-(1-y)^{2} & \text { otherwise. }\end{cases}
$$

In [4], several properties of $(G, N)$-implications were studied. In the following, we present some of them, which are used in this paper: ${ }^{5}$

\footnotetext{
${ }^{5}$ Since grouping functions are disjunctors, see also [37] for the properties of $(A, N)$-implications when $A$ is a disjunctor and $N$ is the standard negation.
} 
Proposition 2.5. [4, Proposition 3.4] Let $G$ and $N$ be a grouping function and a negation, respectively. Then it holds that:

(i) $I_{G, N}$ does not satisfy $(\boldsymbol{O P}){ }^{6}$

(ii) $I_{G, N}$ satisfies $(\mathbf{L O P})$ if and only if $N=N_{\top}$;

(iii) $I_{G, N}$ satisfies $(\mathbf{S B C})(\boldsymbol{a})$ if and only if $N$ satisfies $(\mathbf{N 8})$;

(iv) $I_{G, N}$ satisfies $(\mathbf{S B C})(\boldsymbol{b})$ if and only if $N$ satisfies $(\mathrm{N7})$;

(v) $I_{G, N}$ satisfies $(\mathbf{S B C})$ if and only if $N$ satisfies (N6);

(vi) If $N$ is a strong fuzzy negation then $I_{G, N}$ satisfies $(\boldsymbol{C P})$;

(vii) If $I_{G, N}$ satisfies (CP) for $N$ and 0 is the neutral element of $G$ then $N$ is a strong fuzzy negation;

(viii) If $I_{G, N}$ satisfies (CP) for $N$ and $G$ has a strict section for an element in the range of $N$ then $N$ is a strong fuzzy negation;

(ix) $I_{G, N}$ satisfies (LCP) for $N$ and 0 is the neutral element of $G$ then $N$ is a strong fuzzy negation;

(x) If $N$ is strict and $I_{G, N}$ satisfies $(\boldsymbol{N P})$ and (LCP) then $N_{I_{G, N}}=N^{-1}$;

(xi) If $I_{G, N}$ satisfies $(\mathbf{N P})$ then $N_{I_{G, N}}=N$.

In [4], we provided a characterization of $(G, N)$-implications when $N$ is a strong fuzzy negation.

Theorem 2.2. [4, Theorem 4.1,Theorem 4.2] The following statements hold:

(i) If a function $I:[0,1]^{2} \rightarrow[0,1]$ is continuous and satisfies $(\boldsymbol{I} 2),(\boldsymbol{S B C}),(\boldsymbol{C P})$ and $(\boldsymbol{L C P})$ for the fuzzy negation $N_{I}$ (Definition 2.11), then I is a $(G, N)$-implication.

(ii) If $N:[0,1] \rightarrow[0,1]$ is a strong fuzzy negation, then the $(G, N)$-implication $I_{G, N}:[0,1]^{2} \rightarrow[0,1]$ is continuous and satisfies (I2), (SBC), (CP) and (LCP) for $N_{I_{G, N}}$.

Next corollary, which follows directly from Theorem 2.2, provides a characterization of $(G, N)$-implications when $N$ is a strong fuzzy negation.

Corollary 2.2. [4, Corollary 3.1] Let $N:[0,1] \rightarrow[0,1]$ be a strong fuzzy negation and consider a bivariate function $I:[0,1]^{2} \rightarrow[0,1]$. Then, there exists a grouping function $G:[0,1]^{2} \rightarrow[0,1]$ such that $I=I_{G, N}$ if and only if $I$ is continuous and satisfies (I2), (SBC), (CP) and (LCP) for $N_{I}$.

\subsubsection{QL-implications derived from tuples $(O, G, N)$}

The class of fuzzy implications called $Q L$-implications derived from tuples $(O, G, N)$, where $O, G$ and $N$ denote, respectively, overlap and grouping functions, and fuzzy negations, were introduced by Dimuro et al. in [10]. $Q L-$ implications are special kind of $Q L$-operators, when $N$ is the greatest fuzzy negation (Equation (2)).

Definition 2.12. [10, Definition 3.1] A function $I:[0,1]^{2} \rightarrow[0,1]$ is a $Q L$-operation derived from a tuple $(O, G, N)$ if there exist an overlap function $O:[0,1]^{2} \rightarrow[0,1]$, a grouping function $G:[0,1]^{2} \rightarrow[0,1]$ and a fuzzy negation $N:[0,1] \rightarrow[0,1]$, such that

$$
I(x, y)=G(N(x), O(x, y))
$$

for all $x, y \in[0,1]$. We denote such $Q L$-operation by $I_{O, G, N}$.

\footnotetext{
${ }^{6}$ This is also a direct consequence of [37, Proposition 43].
} 
Observe that, whenever $N$ is a continuous fuzzy negation, then $I_{O, G, N}$ is also continuous.

Theorem 2.3. [10, Theorem 4.1] A $Q L$-operation derived from the tuple $(O, G, N)$ is a fuzzy implication function if and only if $N=N_{\top}$. In this case, the $Q L$-implication derived from the tuple $\left(O, G, N_{\top}\right)$ is given by:

$$
I_{O, G, N_{\top}}(x, y)= \begin{cases}G(0, O(1, y)) & \text { if } x=1 \\ 1 & \text { if } x<1 .\end{cases}
$$

Example 2.5. Consider the overlap function $\mathrm{O}_{2}$ (Equation (5), for $p=2$ ) and its dual grouping function $G_{2}$ (Equation (11)). The $Q L$-implication function derived from $\mathrm{O}_{2}$ and $G_{2}$ is given by [10]:

$$
I_{O_{2}, G_{2}, N_{\top}}(x, y)= \begin{cases}y^{2}\left(2-y^{2}\right) & \text { if } x=1 \\ 1 & \text { if } x<1 .\end{cases}
$$

In [10], several properties of $Q L$-implications were studied. In the following, we present some of them, which are used in this paper:

Proposition 2.6. Let $O:[0,1]^{2} \rightarrow[0,1]$ be an overlap function, $G:[0,1]^{2} \rightarrow[0,1]$ be a grouping function and $N_{\top}:[0,1] \rightarrow[0,1]$ be the greatest fuzzy negation. For a $Q L$-implication $I_{O, G, N_{\top}}:[0,1]^{2} \rightarrow[0,1]$, derived from a tuple $\left(O, G, N_{\mathrm{T}}\right)$, it holds that:

(i) $I_{O, G, N_{\top}}$ does not satisfy $(\boldsymbol{O P})$.

(iii) $I_{O, G, N_{\top}}$ satisfies (LOP).

\section{Introducing $D$-operations and $D$-implications derived from tuples $(O, G, N)$}

In this section, we introduce the concepts of $D$-operations and $D$-implications derived from tuples $(O, G, N)$, denoted by $I_{O, G, N}^{D}$. We show that $I_{O, G, N}^{D}$ are fuzzy implications just in the case when one considers the greatest fuzzy negation $N_{T}$. We also study some properties of $D$-operations and $D$-implications that are necessary for the development of this paper.

Definition 3.1. A function $I:[0,1]^{2} \rightarrow[0,1]$ is a D-operation constructed from tuples $(O, G, N)$ if there exist an overlap function $O:[0,1]^{2} \rightarrow[0,1]$, a grouping function $G:[0,1]^{2} \rightarrow[0,1]$ and a fuzzy negation $N:[0,1] \rightarrow[0,1]$, such that

$$
I(x, y)=G(O(N(x), N(y)), y)
$$

for all $x, y \in[0,1]$. We denote such $D$-operation by $I_{O, G, N}^{D}$, and say that $I_{O, G, N}^{D}$ is a D-operation constructed from the tuple $(O, G, N)$.

Observe that, whenever $N$ is a continuous fuzzy negation, then $I_{O, G, N}^{D}$ is also continuous.

Proposition 3.1. Any D-operation derived from the tuple $\left(O, G, N_{\top}\right)$ is of the form:

$$
I_{O, G, N_{\top}}^{D}(x, y)= \begin{cases}G(0, y) & \text { if } x=1 \wedge y \neq 1 \\ 1 & \text { otherwise. }\end{cases}
$$

ProOF. One has to consider the following cases:

$x=1$ and $y \neq 1$ : In this case, since $N_{\top}(x)=N_{\top}(1)=0$ and $N_{\top}(y)=1$, one has that:

$$
I_{O, G, N_{\top}}^{D}(1, y)=G\left(O\left(N_{\top}(1), N_{\top}(y)\right), y\right)=G(O(0,1), y)=G(0, y) .
$$

$y=1:$ In this case, it is immediate that:

$$
I_{O, G, N_{\top}}^{D}(x, 1)=G\left(O\left(N_{\top}(x), N_{\top}(1)\right), 1\right)=1 .
$$


$x \neq 1$ and $y \neq 1:$ In this case, since $N_{\top}(x)=N_{\top}(y)=1$, one has that:

$$
I_{O, G, N_{\top}}^{D}(x, y)=G\left(O\left(N_{\top}(x), N_{\top}(y)\right), y\right)=G(O(1,1), y)=G(1, y)=1 .
$$

It follows that:

$$
I_{O, G, N_{\top}}^{D}(x, y)= \begin{cases}G(0, y) & \text { if } x=1 \wedge y \neq 1 \\ 1 & \text { otherwise. }\end{cases}
$$

Theorem 3.1. A D-operation derived from a tuple $(O, G, N)$ is a fuzzy implication if and only if $N=N_{\top}$.

Proof. $(\Rightarrow)$ Suppose that $I_{O, G, N}^{D}$ is a fuzzy implication and $N \neq N_{\top}$. Then there exists $\left.y \in\right] 0,1[$ such that $N(y)<1$. Then, since $O(1, N(y))<1$, one has that:

$$
I_{O, G, N}^{D}(0, y)=G(O(N(0), N(y)), y)=G(O(1, N(y)), y)<1,
$$

which is a contradiction, since any fuzzy implication satisfies (LBC). Therefore, one concludes that $N=N_{\top}$.

$(\Leftarrow)$ Suppose that $N=N_{\top}$. Then, by Proposition 3.1, we can prove the properties of fuzzy implications considering $D$-implications given by Equation (23). First observe that (I3) $I_{O, G, N_{\top}}^{D}(0,0)=1$, (I4) $I_{O, G, N_{\top}}^{D}(1,1)=1$ and (I5) $I_{O, G, N_{\top}}^{D}(1,0)=G(0,0)=0$. Now, for all $x, x_{1}, x_{2}, y, y_{1}, y_{2} \in[0,1]$, it follows that:

(I1) Suppose that $x_{1} \leq x_{2}$. The result is immediate for $x_{1}=x_{2}=1$ and $y \neq 1$, or $y=1$. Whenever $x_{1}<1$ and $y<1$, one has that $I_{O, G, N_{\top}}^{D}\left(x_{1}, y\right)=1 \geq I_{O, G, N_{\top}}^{D}\left(x_{2}, y\right)$.

(I2) Suppose that $y_{1} \leq y_{2}$. The result is immediate for $x=1$ and $y_{1}=y_{2}=1$, or $x \neq 1$. Now, if $x=1, y_{1}<1$ and $y_{2}<1$, then one has that $I_{O, G, N_{\top}}^{D}\left(x, y_{1}\right)=G\left(0, y_{1}\right) \leq G\left(0, y_{2}\right)=I_{O, G, N_{\top}}^{D}\left(x, y_{2}\right)$. On the other hand, if $x=1, y_{1}<1$ and $y_{2}=1$, then it holds that $I_{O, G, N_{\top}}^{D}\left(x, y_{1}\right)=G\left(0, y_{1}\right)<1=I_{O, G, N_{\top}}^{D}\left(x, y_{2}\right)$.

Example 3.1. Consider the overlap function $\mathrm{O}_{2}$ (Equation (5), for $p=2$ ) and its dual grouping function $G_{2}$ (Equation (11)). The D-implication function derived from $\mathrm{O}_{2}$ and $\mathrm{G}_{2}$ is given by:

$$
I_{O_{2}, G_{2}, N_{\top}}^{D}(x, y)= \begin{cases}1-(1-y)^{2} & \text { if } x=1 \wedge y \neq 1 \\ 1 & \text { if otherwise. }\end{cases}
$$

Now consider the grouping function

$$
G_{D B}(x, y)= \begin{cases}\frac{x+y-2 x y}{2-(x+y)} & \text { if } x+y \neq 2 \\ 0 & \text { if } x+y=2 .\end{cases}
$$

The D-implication function derived from $\mathrm{O}_{2}$ and $G_{D B}$ is defined by

$$
I_{O_{2}, G_{D B}, N_{\top}}^{D}(x, y)= \begin{cases}\frac{y}{2-y} & \text { if } x=1 \wedge y \neq 1 \\ 1 & \text { otherwise. }\end{cases}
$$

Proposition 3.2. Let $O:[0,1]^{2} \rightarrow[0,1]$ be an overlap function, $G:[0,1]^{2} \rightarrow[0,1]$ be a grouping function and $N:[0,1] \rightarrow[0,1]$ be a fuzzy negation. For a D-operation $I_{O, G, N}^{D}:[0,1]^{2} \rightarrow[0,1]$, derived from a tuple $(O, G, N)$, it holds that:

(i) $I_{O, G, N}^{D}$ does not satisfy $(\boldsymbol{O P})$.

(ii) Whenever $N=N_{\top}$, the D-implication $I_{O, G, N_{\top}}^{D}$ does not satisfy $(\boldsymbol{O P})$. 
(iii) $I_{O, G, N}^{D}$ satisfies $(\mathbf{L O P})$ if and only if $N=N_{\top}$, that is, it is a D-implication.

PROOF. It follows that:

(i) One has that that:

$$
\left.I_{O, G, N}^{D}(x, y)=G(O(N(x), N(y)), y)\right)=1 \Leftrightarrow O(N(x), N(y))=1 \vee y=1 \Leftrightarrow N(x)=N(y)=1 \vee y=1 .
$$

Suppose that there exist $x, y \neq 0$ such that $N(x)=N(y)=1$ and consider $y=\frac{x}{2}$. Observe that $I_{O, G, N}^{D}(x, y)=$ 1 , but $x>y$. Thus, in this case, $I_{O, G, N}^{D}$ does not satisfy (OP). Suppose that there do not exist $x, y \neq 0$ such that $N(x)=N(y)=1$, and take $x=0.5$ and $y=0.7$. Then, one has that $N(x), N(y) \neq 1$ and $y \neq 1$. Therefore, $I_{O, G, N}^{D}(x, y) \neq 1$, that is, $I_{O, G, N}^{D}$ does not satisfy $(\mathbf{O P})$ in this case either.

(ii) It follows from (i).

(iii) $(\Rightarrow)$ Suppose that $N \neq N_{\top}$. Then, there exists $\left.x, y \in\right] 0,1[$ such that $N(x)<1$ or $N(y)<1$. Consider $y>x$. One has that $I_{O, G, N}^{D}(x, y)=G(O(N(x), N(y)), y) \neq 1$, since $O(N(x), N(y))<1$ and $y<1$. Thus, $I_{O, G, N}^{D}$ does not satisfy (LOP). Thus, by contraposition, if $I_{O, G, N}^{D}$ satisfies (LOP) then $N=N_{\top}$.

$(\Leftarrow)$ Suppose that $N=N_{\top}$ and consider $x \leq y$. Then, by Proposition 3.1, considering Equation (23), one has that, for all $x, y \in[0,1], I_{O, G, N_{\top}}(x, y)=1$. In fact, observe that whenever $x=y=1$, or $x<1$ and $y=1$, or $x<1$ and $y<1$, the result holds.

\section{Fuzzy Implications and the $O$-Conditionality}

In this section, we study the law of conditionality for any fuzzy implication $I:[0,1]^{2} \rightarrow[0,1]$ and overlap function $O:[0,1]^{2} \rightarrow[0,1]$, which is a functional inequality that we call by the law of $O$-conditionality. First we define such law, and then we discuss the advantages one may get using it. Finally, several properties are analysed.

Definition 4.1. A fuzzy implication I satisfies the law of $O$-conditionality for an overlap function $O$ if and only if, for all $x, y \in[0,1]$, it holds that:

(OC) $O(x, I(x, y)) \leq y$.

In fact, (OC) means $x *_{O}(x \rightarrow y) \leq y$, and is equivalent to (TC) whenever $T$ is a positive (without zero divisors) and continuous t-norm.

Example 4.1. Observe that the use of $(\mathrm{OC})$ can provide more flexibility and generality in the implementation of the GMP. In fact, let us consider two referential sets $X=\left\{x_{1}, x_{2}\right\}$ and $Y=\left\{y_{1}, y_{2}\right\}$, and the fuzzy rule of the type:

If $x$ is $A$ then $y$ is $B$,

where $A=\left\{\left(x_{1}, A\left(x_{1}\right)\right),\left(x_{2}, A\left(x_{2}\right)\right)\right\}$ and $B=\left\{\left(y_{1}, B\left(y_{1}\right)\right),\left(y_{2}, B\left(y_{2}\right)\right)\right\}$ are fuzzy sets over $X$ and $Y$, respectively. Then, whenever $I:[0,1]^{2} \rightarrow[0,1]$ is an implication function, Rule (26) can be represented by means of the following matrix:

$$
R=\left(\begin{array}{ll}
I\left(A\left(x_{1}\right), B\left(y_{1}\right)\right) & I\left(A\left(x_{1}\right), B\left(y_{2}\right)\right) \\
I\left(A\left(x_{2}\right), B\left(y_{1}\right)\right) & I\left(A\left(x_{2}\right), B\left(y_{2}\right)\right) .
\end{array}\right)
$$

Now, given a fact described in terms of a fuzzy set $A^{\prime}$ over $X$, namely, $A^{\prime}=\left\{\left(x_{1}, A^{\prime}\left(x_{1}\right)\right),\left(x_{2}, A^{\prime}\left(x_{2}\right)\right)\right\}$, and given a $t$-norm $T:[0,1]^{2} \rightarrow[0,1]$, the law of $T$-conditionality amounts to require that:

$$
T\left(A^{\prime}\left(x_{i}\right), I\left(A^{\prime}\left(x_{i}\right), B\left(y_{j}\right)\right)\right) \leq B\left(y_{j}\right),
$$


for $i, j=1,2$. Clearly, in the Inequality (27), the t-norm $T$ is only applied over two variables. This means that associativity is not required, and the $t$-norm $T$ can be replaced by an overlap function $O:[0,1]^{2} \rightarrow[0,1]$. Note that, in particular, since every continuous and positive t-norm is a particular instance of overlap function, if we replace the t-norm $T$ by an overlap function $O$, we are recovering some of the classes of the usual GMP. Furthermore, observe that, for instance, if we consider the overlap function defined, for all $x, y \in[0,1]$ and $p>1$, by:

$$
O(x, y)=(x y)^{p}
$$

and if we take an implication function I such that, for all $x, y \in[0,1]$, it holds that

$$
I(x, y) \leq y
$$

then we have that

$$
O(x, I(x, y))=x^{p}(I(x, y))^{p} \leq x^{p} y^{p} \leq y
$$

and, so, the law of $O$-conditionality holds even if $O$ is not associative, and, hence, the use of overlap functions to implement the GMP provides more generality.

Remark 4.1. In the literature, there are other works on the implementation of the GMP using other conjunctive aggregation functions than $t$-norms. This the case of the law of $U$-conditionality introduced by Mas et al. [55], where $U$ is a uninorm. ${ }^{7}$ In particular, the authors investigated this law for $R U$-implications derived from uninorms [56] and also $(U, N)$-implications based on uninorms and fuzzy negations [57]. However, since uninorms are associative and it is required a neutral element, the U-conditionality, although more general than T-conditionality, presents less generality than $O$-conditionality.

In the following, we state under which conditions of $I$ and $O$ we have that $I$ satisfies (OC) for $O$.

Proposition 4.1. If a fuzzy implication I satisfies (CAB) and (LOP), then I satisfies (OC) for any overlap function O satisfying (06).

Proof. If $I$ satisfies (LOP), then, for all overlap function $O$ satisfying (O6), whenever $x \leq y$, one has that $O(x, I(x, y))=O(x, 1) \leq x \leq y$. On the other hand, since $I$ satisfies (CAB), whenever $x>y$ then, for all overlap function $O$ satisfying (O6), it follows that

$$
O(x, I(x, y)) \leq O(x, y)=O(y, x) \leq O(y, 1) \leq y .
$$

Proposition 4.2. Considering a fuzzy implication $I$, if there exist $x, y \in[0,1]$ such that $x>y$ and $I(x, y)=1$, then I does not satisfy (OC) for any overlap functions $O$ satisfying (07).

Proof. Suppose that there exist $x_{0}, y_{0} \in[0,1]$ such that $x_{0}>y_{0}$ and $I\left(x_{0}, y_{0}\right)=1$. Then, whenever $O$ satisfies (O7), it holds that

$$
O\left(x_{0}, I\left(x_{0}, y_{0}\right)\right)=O\left(x_{0}, 1\right) \geq x_{0}>y_{0} .
$$

Proposition 4.3. Let $I:[0,1]^{2} \rightarrow[0,1]$ be a fuzzy implication function. If I satisfies (OC) for some overlap function $O$, then $N_{I}=N_{\perp}$.

\footnotetext{
${ }^{7}$ A function $U:[0,1]^{2} \rightarrow[0,1]$, is called a uninorm if it fulfills the associativity, commutativity, and increasingness in each place and has a neutral element in $[0,1]$.
} 
Proof. If $x>0$, then $O\left(x, N_{I}(x)\right)=O(x, I(x, 0)) \leq 0$, by (OC). Since $x>0$, then, by (O2), it holds that $N_{I}(x)=0$. Since $N_{I}(0)=1$, one concludes that $N_{I}=N_{\perp}$.

Corollary 4.1. If a fuzzy implication I satisfies (OC) for some overlap function $O$, then $O\left(x, N_{I}(x)\right)=0$.

Proof. If $x=0$, then it is immediate that $O\left(0, N_{I}(0)\right)=0$. On the other hand, if $x>0$, by Proposition 4.3, it holds that

$$
O\left(x, N_{I}(x)\right)=O\left(x, N_{\perp}(x)\right)=O(x, 0)=0 .
$$

Proposition 4.4. If a fuzzy implication I satisfies $(\mathbf{O C})$ for some overlap function $O_{1}:[0,1]^{2} \rightarrow[0,1]$, then I satisfies (OC) for any overlap function $O_{2}:[0,1]^{2} \rightarrow[0,1]$ such that $O_{2} \leq O_{1}$.

Proof. One has that $O_{2}(x, I(x, y)) \leq O_{1}(x, I(x, y)) \leq y$.

Proposition 4.5. If a fuzzy implication $I_{1}:[0,1]^{2} \rightarrow[0,1]$ satisfies (OC) for some overlap function $O$, then any fuzzy implication $I_{2}:[0,1]^{2} \rightarrow[0,1]$ such that $I_{2} \leq I_{1}$ satisfies $(\mathbf{O C})$ for $O$.

Proof. One has that $O\left(x, I_{2}(x, y)\right) \leq O\left(x, I_{1}(x, y)\right) \leq y$.

Proposition 4.6. If two fuzzy implications $I_{1}:[0,1]^{2} \rightarrow[0,1]$ and $I_{2}:[0,1]^{2} \rightarrow[0,1]$ satisfy (OC) for the same overlap function $O$, then the function $\left(I_{1} \vee I_{2}\right):[0,1]^{2} \rightarrow[0,1]$, defined by

$$
\left(I_{1} \vee I_{2}\right)(x, y)=\max \left\{I_{1}(x, y), I_{2}(x, y)\right\}
$$

is a fuzzy implication satisfying (OC) for $O$.

PROOF. Observe that $\left(I_{1} \vee I_{2}\right)$ is a particular case of an aggregation of fuzzy implications, and then it is also a fuzzy implication. If $\left(I_{1} \vee I_{2}\right)(x, y)=I_{1}(x, y)$, then it holds that

$$
O\left(x,\left(I_{1} \vee I_{2}\right)(x, y)\right)=O\left(x, I_{1}(x, y)\right) \leq y .
$$

The proof for $\left(I_{1} \vee I_{2}\right)(x, y)=I_{2}(x, y)$ is analogous.

Now, we analyse the action of an automorphism on a fuzzy implication $I$ satisfying (OC) for some overlap function $O$.

Proposition 4.7. Let $\varphi:[0,1] \rightarrow[0,1]$ be an automorphism. If a fuzzy implication I satisfies (OC) for some overlap function $O$, then $I^{\varphi}:[0,1]^{2} \rightarrow[0,1]$ satisfies $(\mathbf{O C})$ for $O^{\varphi}:[0,1]^{2} \rightarrow[0,1]$.

Proof. Suppose that $I$ satisfies (OC) for some overlap function $O$. Then, it follows that:

$$
\begin{aligned}
O^{\varphi}\left(x, I^{\varphi}(x, y)\right) & =\varphi^{-1}\left(O\left(\varphi(x), \varphi\left(I^{\varphi}(x, y)\right)\right)\right) \\
& =\varphi^{-1}\left(O\left(\varphi(x), \varphi\left(\varphi^{-1}(I(\varphi(x), \varphi(y)))\right)\right)\right) \\
& =\varphi^{-1}(O(\varphi(x), I(\varphi(x), \varphi(y)))) \\
& \leq \varphi^{-1}(\varphi(y)) \\
& =y .
\end{aligned}
$$

\section{The conditional antecedent boundary condition}

In the previous section, we have showed that the conditional antecedent boundary condition (CAB), associated to the left ordering property (LOP), is important for the analysis of the $O$-conditionality of fuzzy implications (see Proposition 4.1). The left ordering property of fuzzy implications derived from overlap and grouping functions was already studied in previous works (see Section 3). In this section, we introduce several results related to the conditional antecedent boundary condition (CAB). 
5.1. (CAB) for $R_{O}$-implications

Theorem 5.1. Let $O:[0,1]^{2} \rightarrow[0,1]$ be an overlap function. $I_{O}$ satisfies $(\boldsymbol{C A B})$ if and only if $O \geq \min$.

PROOF. It follows that:

$(\Rightarrow)$ If $O \nsupseteq \min$, then there exist $x^{\prime}, y^{\prime} \in[0,1]$ such that $O\left(x^{\prime}, y^{\prime}\right)<\min \left\{x^{\prime}, y^{\prime}\right\}$. Now, assume that $x^{\prime} \geq y^{\prime}$. In this case, one has that $O\left(x^{\prime}, y^{\prime}\right)<y^{\prime}$. It holds that

$$
I_{O}\left(x^{\prime}, y^{\prime}\right)=\max \left\{z \in[0,1] \mid O\left(x^{\prime}, z\right) \leq y^{\prime}\right\}>y^{\prime},
$$

which is a contradiction with $(\mathbf{C A B})$. By the commutativity of $O$, if $x^{\prime}<y^{\prime}$, then $I_{O}\left(y^{\prime}, x^{\prime}\right)>x^{\prime}$, which is also a contradiction with (CAB).

$(\Leftarrow)$ Consider $O(x, y) \geq \min \{x, y\}$ and suppose that $x>y$. Then, one has that $O(x, y) \geq y$. It follows that for all $z \in[0,1]$ it holds that if $O(x, z) \leq y$ then $z \leq y$, since $O$ is increasing. One concludes that

$$
I_{O}(x, y)=\max \{z \in[0,1] \mid O(x, z) \leq y\} \leq y .
$$

Example 5.1. Consider the following $R_{O}$-implication functions:

$$
\begin{aligned}
& I_{G D}(x, y)=\left\{\begin{array}{ll}
1 & \text { if } x \leq y, \\
y & \text { if } x>y,
\end{array} \quad\right. \text { (given in Equation (15)) } \\
& I_{O_{m \frac{1}{2}}}(x, y)=\left\{\begin{array}{ll}
1 & \text { if } \sqrt{x} \leq y, \\
y^{2} & \text { if } \sqrt{x}>y,
\end{array} \quad\right. \text { (given in Equation (16)) }
\end{aligned}
$$

which are derived by the Minimum t-norm $T_{M}$ and the overlap function $O_{m \frac{1}{2}}$ (Equation (7)), respectively. Since $O_{m \frac{1}{2}} \geq \min$, then, by Theorem 5.1, both $R_{O}$-implication functions satisfy $(\boldsymbol{C} \boldsymbol{A} \boldsymbol{B})$. In fact, one has that whenever $x>y$, then $I_{G D}(x, y)=y$ and $I_{O_{m \frac{1}{2}}}(x, y)=y^{2} \leq y$. On the other hand, the $R_{O}$-implication function

$$
I_{O_{m M}^{V}}(x, y)= \begin{cases}\min \left\{1, \max \left\{\frac{\sqrt{2 y-1}}{2 \sqrt{2 x-1}}, \frac{2 y-1}{2(2 x-1)^{2}}\right\}+\frac{1}{2}\right\} & \text { if } x \in] 0.5,1] \text { and } y \in[0.5,1], \\ y & \text { if } y \in[0,0.5[\text { and } x>y, \\ 1 & \text { if } x \in[0,0.5] \text { and } x \leq y,\end{cases}
$$

given in Equation (14), which is derived from the overlap function $O_{m M}^{V}$ (Equation (4)), does not satisfy (CAB), by Theorem 5.1, since $O_{m M}^{V}<\min$. In fact, if $x=1$ and $y=0.9$, one has that $I_{O_{m M}^{V}}(1,0.9)=\frac{\sqrt{0.8}+1}{2}>0.9$.

5.2. (CAB) for $(G, N)$-implications

Theorem 5.2. Let $G:[0,1]^{2} \rightarrow[0,1]$ be a grouping function and $N:[0,1] \rightarrow[0,1]$ be a fuzzy negation. Then $I_{G, N}$ satisfies (CAB) if and only if $N=N_{\perp}$ and $G$ satisfies $(\boldsymbol{G 7})$.

ProOF. It follows that:

$\Leftrightarrow)$ Suppose that $I_{G, N}$ satisfies (CAB) and $N \neq N_{\perp}$. Then there exists $x^{\prime}>0$ such that $N\left(x^{\prime}\right) \neq 0$. Then, by (G2), it follows that

$$
I_{G, N}\left(x^{\prime}, 0\right)=G\left(N\left(x^{\prime}\right), 0\right) \neq 0,
$$

that is, $I_{G, N}\left(x^{\prime}, 0\right)>0$, which is a contradiction with $(\mathbf{C A B})$. Now, suppose that $G$ does not satisfy $(\mathbf{G 7})$. Then, for some $y^{\prime} \in\left[0,1\left[\right.\right.$, one has that $G\left(0, y^{\prime}\right)>y^{\prime}$. It follows that

$$
I_{G, N}\left(1, y^{\prime}\right)=G\left(N(1), y^{\prime}\right)=G\left(0, y^{\prime}\right)>y^{\prime},
$$

which is a contradiction with (CAB). Therefore, if $I_{G, N}$ satisfies (CAB) then $N=N_{\perp}$ and $G$ satisfies (G7). $(\Leftarrow)$ Suppose that $N=N_{\perp}$ and that $G$ satisfies (G7). Whenever $x>y$ then, since $x \neq 0$, one has that $N_{\perp}(x)=0$. It follows that

$$
I_{G, N_{\perp}}(x, y)=G\left(N_{\perp}(x), y\right)=G(0, y) \leq y .
$$

Thus, $I_{G, N}$ satisfies $(\mathbf{C A B})$. 
Corollary 5.1. Let $G:[0,1]^{2} \rightarrow[0,1]$ be a grouping function and $N:[0,1] \rightarrow[0,1]$ be a fuzzy negation. If $N$ is not non-filling then the fuzzy implication $I_{G, N}$ does not satisfy $(\boldsymbol{C A B})$.

Proof. If $N$ is not non-filling, then, by (N7), there exists $\left.x^{\prime} \in\right] 0,1\left[\right.$ such that $N\left(x^{\prime}\right)=1$, and, thus $N \neq N_{\perp}$. Then, by Theorem 5.2, $I_{G, N}$ does not satisfy (CAB).

Example 5.2. We illustrate the results of this section, showing three different cases:

(i) Consider the grouping function $G_{M p}(x, y)=\max \left\{x^{p}, y^{p}\right\}$, with $p \geq 1$ (Equation (10)), which obviously satisfies (G7), and the least fuzzy negation $N_{\perp}$ given in Equation (1), which generate the $(G, N)$-implication function

$$
I_{G_{M p}, N_{\perp}}(x, y)= \begin{cases}1 & \text { if } x=0, \\ y^{p} & \text { otherwise }\end{cases}
$$

given in Equation (19). By Theorem 5.2, it holds that $I_{G_{M p}, N_{\perp}}$ satisfies $(\boldsymbol{C A B})$. In fact, whenever $x>y$, then $x \neq 0$ and

$$
I_{G_{M p}, N_{\perp}}(x, y)=y^{p} \leq y .
$$

(ii) Consider the grouping function $G_{M 2}(x, y)=\max \left\{x^{2}, y^{2}\right\}$ (Equation (10) for $p=2$ ), which obviously satisfies (G7), and the fuzzy negation $N_{3}(x)=\sqrt{1-x}$, which generate the $(G, N)$-implication

$$
I_{G_{M 2}, N_{3}}(x, y)=\max \left\{1-x, y^{2}\right\} \text { (Equation (18)). }
$$

By Theorem 5.2, it holds that $I_{G_{M 2}, N_{3}}$ does not satisfy $(\boldsymbol{C A B})$. In fact, considering $y=0$ and $0<x<1$, one has that:

$$
I_{G_{M 2}, N_{3}}(x, 0)=\max \{1-x, 0\}=1-x>0=y .
$$

Let us now analyse the $(G, N)$-implication function

$$
I_{G_{M 2}, N}(x, y)=\max \left\{N(x)^{2}, y^{2}\right\},
$$

considering any fuzzy negation $N:[0,1] \rightarrow[0,1]$. Again, whenever one considers the case of $y=0$ and $0<x<1$, one has that whenever $I_{G_{M 2}, N}$ satisfies $(\boldsymbol{C A B})$, then, since $x>y$ it holds that

$$
I_{G_{M 2}, N}(x, 0)=\max \left\{N(x)^{2}, 0\right\}=N(x)^{2} \leq 0,
$$

which implies that $N(x)=0$ for all $x \in] 0,1\left[\right.$, that is, it implies that $N=N_{\perp}$, in accordance with Theorem 5.2.

(iii) Consider the grouping function $G_{2}(x, y)=1-(1-x)^{2}(1-y)^{2}$ (Equation (11)). Observe that $G 2$ does not satisfy (G7), since, for $y=0.9$, one has that $G_{2}(0,0.9)=1-(1-0.9)^{2}>0.9=y$. The grouping function $G_{2}$ and the least fuzzy negation $N_{\text {bot }}$ (Equation (1)) generate the $(G, N)$-implication function

$$
I_{G_{2}, N_{\perp}}(x, y)= \begin{cases}1 & \text { if } x=0, \\ 1-(1-y)^{2} & \text { otherwise, }\end{cases}
$$

given in Equation (20). By Theorem 5.2, it holds that $I_{G_{2}, N_{\perp}}$ does not satisfy (CAB). In fact, whenever $x=1$ and $y=0.1$, one has that:

$$
I_{G_{2}, N_{\perp}}(1,0.1)=0.19>0.1=y .
$$




\section{3. (CAB) for $Q L$ - and D-Implications derived from tuples $\left(O, G, N_{T}\right)$}

Unfortunately, there is no $Q L$-implication functions derived from tuples $\left(O, G, N_{\top}\right)$ satisfying $(\boldsymbol{C A B})$. Similarly, there is no $D$-implication functions satisfying this property.

Theorem 5.3. Let $O:[0,1]^{2} \rightarrow[0,1]$ be an overlap function, $G:[0,1]^{2} \rightarrow[0,1]$ be a grouping function and $N_{\top}:[0,1] \rightarrow[0,1]$ be the greatest fuzzy negation defined by Equation (2). Then, any $Q L$-implication $I_{O, G, N_{\top}}$ : $[0,1]^{2} \rightarrow[0,1]$ does not satisfy $(\mathbf{C A B})$.

Proof. Take $x, y \in\left[0,1\left[\right.\right.$ such that $1>x>y$. Then, by Equation (21), one has that $I_{O, G, N_{\top}}=1>y$, and, thus, $I_{O, G, N_{\top}}$ does not satisfy $(\boldsymbol{C A B})$.

Theorem 5.4. Let $O:[0,1]^{2} \rightarrow[0,1]$ be an overlap function, $G:[0,1]^{2} \rightarrow[0,1]$ be a grouping function and $N_{\top}:[0,1] \rightarrow[0,1]$ be the greatest fuzzy negation defined by Equation (2). Then, any D-implication $I_{O, G, N_{\top}}^{D}$ : $[0,1]^{2} \rightarrow[0,1]$ does not satisfy $(\mathbf{C A B})$.

Proof. Take $x, y \in\left[0,1\left[\right.\right.$ such that $1>x>y$. Then, by Equation (23), one has that $I_{O, G, N_{\top}}^{D}=1>y$, and, thus, $I_{O, G, N_{\top}}^{D}$ does not satisfy $(\boldsymbol{C A B})$.

\section{Fuzzy implications derived from overlap and grouping functions and the $O$-Conditionality}

In this section, we introduce an analysis of the $O$-Conditionality considering the particular cases of fuzzy implications derived from overlap and grouping functions.

\subsection{O-Conditionality for $R_{O}$-Implications}

In this section, we analyse the law of $O$-conditionality for $R_{O}$-implications. In particular, we state under which conditions of the overlap functions $O_{1}$ and $O_{2}$, and of the $R_{O}$-implication $I_{O_{2}}$, we have that $I_{O_{2}}$ satisfies or not the law of $O$-conditionality for $O_{1}$ and/or $O_{2}$.

Proposition 6.1. Let $O_{1}:[0,1]^{2} \rightarrow[0,1]$ be an overlap function satisfying (O6) and $O_{2}:[0,1]^{2} \rightarrow[0,1]$ be an overlap function such that $O_{2} \geq$ min. Then, the $R_{O^{-i m p l i c a t i o n}} I_{O_{2}}:[0,1]^{2} \rightarrow[0,1]$ satisfies $(\mathbf{O C})$ for $O_{1}$.

Proof. If $O_{2} \geq \mathrm{min}$, then, by Proposition 2.2 (vi), it holds that $I_{O_{2}}$ satisfies (CAB), that is, if $x>y$ then $I_{O_{2}}(x, y) \leq$ $y$. On the other hand, by Lemma 2.1(ii), it holds that $O_{1}(x, y) \leq \min \{x, y\} \leq y$, and then, since $O_{1}$ is increasing, one has that $O_{1}\left(x, I_{O_{2}}(x, y)\right) \leq O_{1}(x, y) \leq y$. Now, if $x \leq y$, since $O_{1}$ satisfies (O6) and is increasing, then, $O_{1}\left(x, I_{O_{2}}(x, y)\right) \leq O_{1}(x, 1) \leq x \leq y$.

Corollary 6.1. The following statements hold:

(i) If $\mathrm{O}_{2}:[0,1]^{2} \rightarrow[0,1]$ is an overlap function such that $O_{2} \geq \min$, then $I_{O_{2}}:[0,1]^{2} \rightarrow[0,1]$ satisfies $(\mathbf{O C})$ for any positive continuous $t$-norm $T:[0,1]^{2} \rightarrow[0,1]$.

(ii) $I_{\min }$ satisfies $(\boldsymbol{O C})$ for any overlap function $O:[0,1]^{2} \rightarrow[0,1]$ satisfying (O6).

(iii) $I_{\min }$ satisfies $(\boldsymbol{O C})$ for any positive continuous t-norm $T:[0,1]^{2} \rightarrow[0,1]$.

PROOF. They follow from Proposition 6.1.

Proposition 6.2. Let $O_{1}, O_{2}:[0,1]^{2} \rightarrow[0,1]$ be overlap functions. If there exists $x^{\prime} \in[0,1]$ such that $O_{2}\left(x^{\prime}, 1\right)<x^{\prime}$ and $O_{1}\left(x^{\prime}, 1\right)>x^{\prime}$, then the $R_{O^{-}}$-implication $I_{O_{2}}:[0,1]^{2} \rightarrow[0,1]$ does not satisfy (OC) for $O_{1}$.

Proof. Consider that $I_{O_{2}}$ satisfies (OC) for $O_{1}$. Suppose that there exists $x^{\prime} \in[0,1]$ such that $O_{2}\left(x^{\prime}, 1\right)<x^{\prime}$ and $O_{1}\left(x^{\prime}, 1\right)>x^{\prime}$. It follows that

$$
O_{1}\left(x^{\prime}, I_{O_{2}}\left(x^{\prime}, x^{\prime}\right)\right)=O_{1}\left(x^{\prime}, \sup \left\{z \in[0,1] \mid O_{2}\left(x^{\prime}, z\right) \leq x^{\prime}\right\}\right)=O_{1}\left(x^{\prime}, 1\right)>x^{\prime} .
$$

Thus, $I_{\mathrm{O}_{2}}$ does not satisfy $(\boldsymbol{O C})$ for $O_{1}$. 
Theorem 6.1. Any $R_{O}$-implication $I_{O}:[0,1]^{2} \rightarrow[0,1]$ derived from the overlap function $O:[0,1]^{2} \rightarrow[0,1]$ satisfies $($ OC) for $O$.

Proof. By Proposition 2.1, $O$ and $I_{O}$ form an adjoint pair, that is, they satisfy the residuation property: $\forall x, y, u \in$ $[0,1]: O(x, u) \leq y \Leftrightarrow I_{O}(x, y) \geq u$. Consider $u=I_{O}(x, y)$. Then, it follows that $O\left(x, I_{O}(x, y)\right) \leq y$.

Corollary 6.2. Let $O_{1}, O_{2}:[0,1]^{2} \rightarrow[0,1]$ be overlap functions. If $O_{1} \leq O_{2}$ then $I_{O_{2}}$ satisfies $(\boldsymbol{O C})$ for $O_{1}$.

Proof. From Theorem 6.1, it holds that $I_{O_{1}}$ satisfies (OC) for $O_{1}$. On the other hand, by Proposition 2.3, one has that if $O_{1} \leq O_{2}$ then $I_{O_{2}} \leq I_{O_{1}}$. It follows that

$$
O_{1}\left(x, I_{O_{2}}(x, y)\right) \leq O_{1}\left(x, I_{O_{1}}(x, y)\right) \leq y .
$$

Corollary 6.3. Let $O_{1}, O_{2}:[0,1]^{2} \rightarrow[0,1]$ be overlap functions. If $I_{O_{2}} \leq I_{O_{1}}$ then $I_{O_{2}}$ satisfies $(\boldsymbol{O C})$ for $O_{1}$.

Proof. By Proposition 2.3, one has that if $I_{O_{2}} \leq I_{O_{1}}$ then $O_{1} \leq O_{2}$. By Corollary 6.2, $I_{O_{2}}$ satisfies (OC) for $O_{1}$.

Proposition 6.3. Let $I_{1}, I_{2}:[0,1]^{2} \rightarrow[0,1]$ be fuzzy implications. Whenever $O_{I_{2}}$ is an overlap function, if $I_{1} \leq I_{2}$ then $I_{1}$ satisfies $(\mathbf{O C})$ for $O_{I_{2}}$.

Proof. Whenever $I_{1} \leq I_{2}$, then one has that

$$
O_{I_{2}}\left(x, I_{1}(x, y)\right)=\inf \left\{z \in[0,1] \mid I_{2}(x, z) \geq I_{1}(x, y)\right\} \leq y .
$$

Example 6.1. We analyse the O-conditionality for the following cases:

(i) Consider the overlap function $O_{p}(x, y)=(x y)^{p}$, with $p \geq 1$ (Equation (5)), which obviously satisfies (O6), and the following $R_{O}$-implication functions:

$$
\begin{aligned}
I_{G D}(x, y) & = \begin{cases}1 & \text { if } x \leq y, \\
y & \text { if } x>y ;\end{cases} \\
I_{O_{m \frac{1}{2}}}(x, y) & = \begin{cases}1 & \text { if } \sqrt{x} \leq y, \\
y^{2} & \text { if } \sqrt{x}>y ;\end{cases}
\end{aligned}
$$

given, respectively, in Equations (15) and (16), which are derived by the Minimum t-norm $T_{M}$ and the overlap function $O_{m \frac{1}{2}}$ (Equation (7)). Observe that $O_{m \frac{1}{2}} \geq \min$. Then, by Proposition 6.1, both $I_{G D}$ and $I_{O_{m \frac{1}{2}}}$ satisfy the law of $O$-conditionality for $O_{p}$. In fact, for all $x, y \in[0,1]$, such that $x \leq y$ one has that:

$$
O_{p}\left(x, I_{G D}(x, y)\right)=O_{p}(x, 1)=x^{p} \leq y .
$$

On the other hand, whenever $x>y$, it holds that:

$$
O_{p}\left(x, I_{G D}(x, y)\right)=O_{p}(x, y)=(x y)^{p} \leq y .
$$

(ii) Consider the overlap function $O_{m M}^{V}$

$$
O_{m M}^{V}(x, y)= \begin{cases}\frac{1+O_{m M}(2 x-1,2 y-1)}{2} & \text { if } x, y \in] 0.5,1] \\ \min \{x, y\} & \text { otherwise, }\end{cases}
$$

given in Equation (4) where $O_{m M}(x, y)=\min \{x, y\} \max \left\{x^{2}, y^{2}\right\}$, and the $R_{O}$-implication function

$$
I_{O_{m M}^{V}}(x, y)= \begin{cases}\min \left\{1, \max \left\{\frac{\sqrt{2 y-1}}{2 \sqrt{2 x-1}}, \frac{2 y-1}{2(2 x-1)^{2}}\right\}+\frac{1}{2}\right\} & \text { if } x \in] 0.5,1] \text { and } y \in[0.5,1] \\ y & \text { if } y \in[0,0.5[\text { and } x>y \\ 1 & \text { if } x \in[0,0.5] \text { and } x \leq y\end{cases}
$$


derived from the overlap function $O_{m M}^{V}$. Observe that $O_{m M}^{V}$ satisfies (O6), since, whenever $\left.\left.x \in\right] 0.5,1\right]$, one has that

$$
O_{m M}^{V}(x, 1)=\frac{1+O_{m M}(2 x-1,1)}{2}=\frac{1+\min \{2 x-1,1\} \max \left\{(2 x-1)^{2}, 1\right\}}{2}=x,
$$

and, if $x \in[0,0.5]$, then

$$
O_{m M}^{V}(x, 1)=\min \{x, 1\}=x .
$$

However, $O_{m M}^{V}<\min$, and, thus, Proposition 6.1 is not applicable for the study of the O-conditionality of the $R_{O}$-implication function $I_{O_{m M}^{V}}$ for $O_{m M}^{V}$. Nevertheless, by Theorem 6.1, $I_{O_{m M}^{V}}$ do satisfy the law of $O$-conditionality for $O_{m M}^{V}$.

\subsection{O-Conditionality for $(G, N)$-implications}

In this section, we analyse the law of $O$-conditionality for $(G, N)$-implications. In particular, we state under which conditions of the grouping function $G$, the fuzzy negation $N$, the overlap function $O$, and of the $(G, N)$-implication $I_{G, N}$, we have that $I_{G, N}$ satisfies or not the law of $O$-conditionality for $O$.

Theorem 6.2. Let $O:[0,1]^{2} \rightarrow[0,1]$ be an overlap function, $G:[0,1]^{2} \rightarrow[0,1]$ be a grouping function and $N:[0,1] \rightarrow[0,1]$ be a fuzzy negation. If the $(G, N)$-implication $I_{G, N}:[0,1]^{2} \rightarrow[0,1]$ satisfies $(\mathbf{O C})$ for $O$ then $N=N_{\perp}$.

Proof. Consider that $I_{G, N}$ satisfies (OC) for $O$ and $N \neq N_{\perp}$. Then there exists $\left.x^{\prime} \in\right] 0,1\left[\right.$ such that $N\left(x^{\prime}\right) \neq 0$. Then, by (G2), it holds that $G\left(N\left(x^{\prime}\right), 0\right) \neq 0$, and, thus, by (O2), it follows that:

$$
O\left(x^{\prime}, I_{G, N}\left(x^{\prime}, 0\right)\right)=O\left(x^{\prime}, G\left(N\left(x^{\prime}\right), 0\right)\right)>0,
$$

which is a contradiction with (OC). Thus, one has that $N=N_{\perp}$.

Theorem 6.3. Let $O:[0,1]^{2} \rightarrow[0,1]$ be an overlap function, $G:[0,1]^{2} \rightarrow[0,1]$ be a grouping function, and $N:[0,1] \rightarrow[0,1]$ be a fuzzy negation. If $O$ satisfies $(\mathbf{O 6}), G$ satisfies $(\mathbf{G 7})$ and $N=N_{\perp}$ then the $(G, N)$-implication $I_{G, N_{\perp}}:[0,1]^{2} \rightarrow[0,1]$ satisfies $(\mathbf{O C})$ for $O$.

Proof. If $N=N_{\perp}$, then one has the following cases:

(i) If $x=0$ then $O\left(0, I_{G, N_{\perp}}(0, y)\right)=0 \leq y$.

(ii) If $x>0$ then, since $O$ satisfies (O6), it follows that:

$$
\begin{aligned}
O\left(x, I_{G, N_{\perp}}(x, y)\right) & =O\left(x, G\left(N_{\perp}(x), y\right)\right) \\
& =O(x, G(0, y)) \\
\leq & O(x, y) \text { by }(\mathbf{G} 7) \\
\leq & y \text { by Lemma } 2.1(\mathbf{i}) .
\end{aligned}
$$

Then, $I_{G, N}$ satisfies $(\mathbf{O C})$ for $O$.

Example 6.2. We illustrate the theorems of this section, showing three different cases:

(i) Consider the overlap function $O_{p_{1}}(x, y)=(x y)^{p_{1}}$, with $p_{1} \geq 1$ (Equation (5)), which satisfies trivially (O6). Take the grouping function $G_{M p_{2}}(x, y)=\max \left\{x^{p_{2}}, y^{p_{2}}\right\}$, with $p_{2} \geq 1$ (Equation (10)), which obviously satisfies (G7), and the least fuzzy negation $N_{\perp}$ given in Equation (1), which generate the $(G, N)$-implication function

$$
I_{G_{M p_{2}}, N_{\perp}}(x, y)= \begin{cases}1 & \text { if } x=0, \\ y^{p_{2}} & \text { otherwise },\end{cases}
$$


given in Equation (19). By Theorem 6.3, it holds that $I_{G_{M p_{2}}, N_{\perp}}$ satisfies the law of $O$-conditionality for $O_{p_{1}}$. In fact, for $x=0$, it follows that

$$
O_{p_{1}}\left(0, I_{G_{M p_{2}}, N_{\perp}}(0, y)\right)=O_{p_{1}}(0,1)=0 \leq y .
$$

On the other hand, if $x>0$, then

$$
O_{p_{1}}\left(x, I_{G_{M p_{2}}, N_{\perp}}(x, y)\right)=O_{p_{1}}\left(x, y^{p_{2}}\right)=x^{p_{1}} y^{\left(p_{1} p_{2}\right)} \leq y,
$$

which illustrate the result given by Theorem 6.3 .

(ii) Consider the overlap function $\mathrm{O}_{2}(x, y)=(x y)^{2}$, (Equation (5) for $\left.p=2\right)$, which satisfies trivially (O6). Take the grouping function $G_{M 2}(x, y)=\max \left\{x^{2}, y^{2}\right\}$ (Equation (10) for $p=2$ ), which obviously satisfies (G7), and the fuzzy negation $N_{3}(x)=\sqrt{1-x}$, which generate the $(G, N)$-implication

$$
I_{G_{M 2}, N_{3}}(x, y)=\max \left\{1-x, y^{2}\right\}(\text { Equation (18)). }
$$

By Theorem 6.3, it holds that $I_{G_{M 2}, N_{3}}$ does not satisfy the law of $\mathrm{O}$-conditionality for $\mathrm{O}_{2}$. In fact, considering $y=0$ and $0<x<1$, one has that:

$$
O_{2}\left(x, I_{G_{M 2}, N_{3}}(x, 0)\right)=x^{2}(1-x)^{2}>0=y .
$$

Let us now analyse the $(G, N)$-implication function

$$
I_{G_{M 2}, N}(x, y)=\max \left\{N(x), y^{2}\right\},
$$

defined for any fuzzy negation $N$. Again, whenever one considers the overlap function $\mathrm{O}_{2}$ and the critic case of $y=0$ and $0<x<1$, one has that whenever $I_{G_{M 2}, N}$ satisfies the law of $O$-conditionality for $\mathrm{O}_{2}$, then it holds that

$$
O_{2}\left(x, I_{G_{M 2}, N}(x, 0)\right)=x^{2}(\max \{N(x), 0\})^{2}=x^{2} N(x)^{2} \leq 0,
$$

which implies that $N(x)=0$ for all $x \in] 0,1\left[\right.$, that is, it implies that $N=N_{\perp}$, in accordance with Theorem 6.2 .

(iii) Consider the overlap function $O_{p}(x, y)=(x y)^{p}$, with $p \geq 1$ (Equation (5)), which satisfies trivially (O6), and the grouping function $G_{2}(x, y)=1-(1-x)^{2}(1-y)^{2}$ (Equation (11)). By Example 5.2(iii) G2 does not satisfy (G7). The grouping function $G_{2}$ and the least fuzzy negation $N_{\text {bot }}$ (Equation (1)) generate the $(G, N)$ implication function

$$
I_{G_{2}, N_{\perp}}(x, y)= \begin{cases}1 & \text { if } x=0, \\ 1-(1-y)^{2} & \text { otherwise },\end{cases}
$$

given in Equation (20). By Theorem 6.3, it holds that $I_{G_{2}, N_{\perp}}$ does not satisfy the law of O-conditionality for $O_{p}$. In fact, whenever $x=1$ and $y=0.1$, one has that:

$$
O_{p}\left(1,1-(1-0.1)^{2}\right)=0.19^{p}>0.1=y
$$

6.3. O-Conditionality for $Q L$ - and D-implication functions derived from tuples $\left(O, G, N_{\top}\right)$

In this section, we analyse the law of $O$-conditionality for $Q L$-implication and $D$-implication functions, showing that, as expected, they do not satisfy that law.

Theorem 6.4. Let $O_{1}, O_{2}:[0,1]^{2} \rightarrow[0,1]$ be overlap functions, $G:[0,1]^{2} \rightarrow[0,1]$ be a grouping function and $N_{\top}:[0,1] \rightarrow[0,1]$ be the greatest fuzzy negation defined by Equation (2). Then, any $Q L$-implication $I_{O_{2}, G, N_{\top}}:$ $[0,1]^{2} \rightarrow[0,1]$ does not satisfy $(\mathbf{O C})$ for $\mathrm{O}_{2}$. 
Proof. Take $x \in] 0,1\left[\right.$ and $y=0$. Then, by Equation (21), one has that $I_{O_{2}, G, N_{\top}}(x, y)=1$, and, thus,

$$
O_{1}\left(x, I_{O_{2}, G, N_{\top}}(x, 0)\right)=O_{1}(x, 1)>0=y .
$$

Therefore, $I_{O_{2}, G, N_{\top}}$ does not satisfy (OC).

Theorem 6.5. Let $O_{1}, O_{2}:[0,1]^{2} \rightarrow[0,1]$ be overlap functions, $G:[0,1]^{2} \rightarrow[0,1]$ be a grouping function and $N_{\top}:[0,1] \rightarrow[0,1]$ be the greatest fuzzy negation defined by Equation (2). Then, any D-implication $I_{\mathrm{O}_{2}, G, N_{\top}}^{D}:$ $[0,1]^{2} \rightarrow[0,1]$ does not satisfy (OC) for $\mathrm{O}_{2}$.

Proof. Take $x \in] 0,1\left[\right.$ and $y=0$. Then, by Equation (23), one has that $I_{O_{2}, G, N_{\top}}^{D}(x, y)=1$, and, thus,

$$
O_{1}\left(x, I_{O_{2}, G, N_{\top}}^{D}(x, 0)\right)=O_{1}(x, 1)>0=y .
$$

Therefore, $I_{O_{2}, G, N_{\top}}^{D}$ does not satisfy (OC).

\section{Conclusion}

In general, aggregation functions can be used not only to build implication functions from aggregation functions, but also the opposite direction [37]. On the other hand, several definitions of fuzzy implications, based on aggregation functions other than t-norms and t-conorms, have been introduced in the literature. Depending on the chosen operator, the derived fuzzy implication may or not satisfy certain properties. Nevertheless, there are some properties that may be not demanded for certain applications.

Overlap and grouping functions are a special kind of not necessarily associative bivariate aggregation operators used, in general, in applications involving the overlapping problem and/or when the associativity property is not strongly required, as in image processing and decision making based on fuzzy preference relations, respectively. In those applications, there is no need the use of t-norms/t-conorms as combination operators.

When considering fuzzy implications derived from overlap and grouping functions, certain properties may not be verified, as the exchange or the left neutrality principles, but only weaker versions of these properties.

The present paper presents an analysis of the $O$-Conditionality for fuzzy implications in general, and, in particular, for $R_{O^{-}},(G, N)$-, $Q L$ - and $D$-implications derived from tuples $(O, G, N)$, the latter also introduced in this paper. We also study the conditional boundary condition for such fuzzy implications, since this property, associated to the left ordering property, plays an important role in the analysis of the $O$-Conditionality.

Future theoretical work is concerned with the investigation of the law of $O$-conditionality in the interval-valued setting, as in $[24,46,58]$.

We are also aiming at applications in the context of hybrid BDI-fuzzy [59] ${ }^{8}$ agent models, commonly used in social simulation $[61,62]$, where the evaluation of social values and exchanges are of a qualitative, subjective, vague nature [63, 64]. Overlap functions and grouping functions can be used for dealing with indifference and incomparability when reasoning on the agent's fuzzy belief base, where a kind of weak preference relation may be defined. Fuzzy implications derived from overlap and grouping functions can be used for performing inferences, decision making and in the fuzzy control of the agents's intentions.

\section{Acknowledgments}

This work was partially supported by the Spanish Ministry of Science and Technology under the project TIN201677356-P (AEI/FEDER, UE), and by the Brazilian funding agency CNPQ under Processes 305882/2016-3, 481283/20137, 306970/2013-9, 232827/2014-1 and 307681/2012-2.

[1] M. Baczyński, B. Jayaram, Fuzzy Implications, Studies in Fuzziness and Soft Computing, vol. 231, Springer, Berlin Heidelberg, 2008.

[2] M. Baczyński, On the applications of fuzzy implication functions, in: V. E. Balas, J. Fodor, A. R. Várkonyi-Kóczy, J. Dombi, L. C. Jain (Eds.), Soft Computing Applications, Vol. 195 of Advances in Intelligent Systems and Computing, Springer, Berlin, 2013, pp. 9-10.

\footnotetext{
${ }^{8}$ BDI stands for "Beliefs, Desires, Intentions", a particular cognitive agent model introduced in [60].
} 
[3] M. Baczyński, G. Beliakov, H. Bustince Sola, A. Pradera (Eds.), Advances in Fuzzy Implication Functions, Vol. 300 of Studies in Fuzziness and Soft Computing, Springer, Berlin, 2013.

[4] G. P. Dimuro, B. Bedregal, R. H. N. Santiago, On $(G, N)$-implications derived from grouping functions, Information Sciences 279 (2014) 1 $-17$.

[5] L. A. Zadeh, Outline of a new approach to the analysis of complex systems and decision processes, Systems, Man and Cybernetics, IEEE Transactions on SMC-3 (1) (1973) 28-44.

[6] M. Luo, K. Zhou, Logical foundation of the quintuple implication inference methods, International Journal of Approximate Reasoning 101 (2018) $1-9$.

[7] W. Pedrycz, Fuzzy control and fuzzy systems, technical Report 8214 (1982).

[8] W. Pedrycz, On generalized fuzzy relational equations and their applications, Journal of Mathematics and Analysis and Applications 107 (1985) 520-536.

[9] M. Mas, M. Monserrat, D. Ruiz-Aguilera, J. Torrens, RU and (U,N)-implications satisfying Modus Ponens, International Journal of Approximate Reasoning 73 (2016) 123 - 137.

[10] G. P. Dimuro, B. Bedregal, H. Bustince, A. Jurio, M. Baczyński, K. Miś, QL-operations and QL-implication functions constructed from tuples $(\mathrm{O}, \mathrm{G}, \mathrm{N})$ and the generation of fuzzy subsethood and entropy measures, International Journal of Approximate Reasoning 82 (2017) 170 $-192$.

[11] G. Lucca, G. P. Dimuro, V. Mattos, B. Bedregal, H. Bustince, J. A. Sanz, A family of Choquet-based non-associative aggregation functions for application in fuzzy rule-based classification systems, in: 2015 IEEE International Conference on Fuzzy Systems (FUZZ-IEEE), IEEE, Los Alamitos, 2015, pp. 1-8. doi:10.1109/FUZZ-IEEE.2015.7337911.

[12] H. Bustince, J. Fernandez, R. Mesiar, J. Montero, R. Orduna, Overlap functions, Nonlinear Analysis: Theory, Methods \& Applications 72 (3-4) (2010) 1488-1499.

[13] J. C. Fodor, T. Keresztfalvi, Nonstandard conjunctions and implications in fuzzy logic, International Journal of Approximate Reasoning 12 (2) (1995) $69-84$

[14] M. Elkano, M. Galar, J. Sanz, H. Bustince, Fuzzy rule-based classification systems for multi-class problems using binary decomposition strategies: On the influence of n-dimensional overlap functions in the fuzzy reasoning method, Information Sciences 332 (2016) $94-114$.

[15] G. Beliakov, S. James, T. Calvo, Aggregating fuzzy implications to measure group consensus, in: Proceedings of 2013 Joint IFSA World Congress and NAFIPS Annual Meeting (IFSA/NAFIPS), Los Alamitos, Los Alamitos, 2013, pp. 1016-1021.

[16] G. Beliakov, T. Calvo, S. James, Consensus measures constructed from aggregation functions and fuzzy implications, Knowledge-Based Systems 55 (2014) $1-8$.

[17] D. Paternain, A. Jurio, E. Barrenechea, H. Bustince, B. Bedregal, E. Szmidt, An alternative to fuzzy methods in decision-making problems, Expert Systems with Applications 39 (9) (2012) 7729 - 7735.

[18] Y. Shi, B. V. Gasse, E. Kerre, The role a fuzzy implication plays in a multi-criteria decision algorithm, International Journal of General Systems 42 (1) (2013) 111-120.

[19] B. C. Bedregal, G. P. Dimuro, H. Bustince, E. Barrenechea, New results on overlap and grouping functions, Information Sciences 249 (2013) $148-170$.

[20] G. P. Dimuro, B. Bedregal, H. Bustince, M. J. Asiáin, R. Mesiar, On additive generators of overlap functions, Fuzzy Sets and Systems 287 (2016) 76 - 96, theme: Aggregation Operations.

[21] G. P. Dimuro, B. Bedregal, On residual implications derived from overlap functions, Information Sciences 312 (2015) 78 - 88.

[22] J. Qiao, B. Q. Hu, On interval additive generators of interval overlap functions and interval grouping functions, Fuzzy Sets and Systems 323 (2017) 19 - 55, theme: Aggregation Functions.

[23] J. Qiao, B. Q. Hu, On generalized migrativity property for overlap functions, Fuzzy Sets and Systems(in press, corrected proof online). doi:https://doi.org/10.1016/j.fss.2018.01.007.

[24] M. Cao, B. Q. Hu, J. Qiao, On interval (G,N)-implications and (O,G,N)-implications derived from interval overlap and grouping functions, International Journal of Approximate Reasoning 100 (2018) 135 - 160.

[25] J. Qiao, B. Q. Hu, The distributive laws of fuzzy implications over overlap and grouping functions, Information Sciences 438 (2018) 107 126. doi:https://doi.org/10.1016/j.ins.2018.01.047.

[26] J. Qiao, B. Q. Hu, On homogeneous, quasi-homogeneous and pseudo-homogeneous overlap and grouping functions, Fuzzy Sets and Systems(in press, corrected proof). doi:https://doi.org/10.1016/j.fss.2018.06.001.

[27] J. Barzilai, Consistency measures for pairwise comparison matrices, Journal of Multi-Criteria Decision Analysis 7 (3) (1998) $123-132$.

[28] M. Fedrizzi, S. Giove, Incomplete pairwise comparison and consistency optimization, European Journal of Operational Research 183 (1) (2007) 303 - 313. doi:10.1016/j.ejor.2006.09.065.

[29] F. Chiclana, F. Herrera, E. Herrera-Viedma, Integrating multiplicative preference relations in a multipurpose decision-making model based on fuzzy preference relations, Fuzzy Sets and Systems 122 (2) (2001) 277 - 291.

[30] W. Pedrycz, M. Song, Analytic hierarchy process (AHP) in group decision making and its optimization with an allocation of information granularity, IEEE Transactions on Fuzzy Systems 19 (3) (2011) 527 -539. doi:10.1109/TFUZZ.2011.2116029.

[31] R. O. Parreiras, P. Ekel, F. Bernardes Jr., A dynamic consensus scheme based on a nonreciprocal fuzzy preference relation modeling, Information Sciences 211 (2012) 1 - 17. doi:10.1016/j.ins.2012.05.001.

[32] T.-C. Wang, Y.-H. Chen, Applying fuzzy linguistic preference relations to the improvement of consistency of fuzzy AHP, Information Sciences 178 (19) (2008) 3755 - 3765. doi:10.1016/j.ins.2008.05.028.

[33] J. Fodor, M. Roubens, Fuzzy Preference Modelling and Multicriteria Decision Support, Kluwer Academic Publisher, Dordrecht, 1994.

[34] M. Roubens, P. Vincke, Preference Modeling, no. 250 in Lecture Notes in Economics and Mathematical Systems, Springer, Berlin, 1985.

[35] H. Bustince, M. Pagola, R. Mesiar, E. Hüllermeier, F. Herrera, Grouping, overlaps, and generalized bientropic functions for fuzzy modeling of pairwise comparisons, IEEE Transactions on Fuzzy Systems 20 (3) (2012) 405-415.

[36] Y. Ouyang, On fuzzy implications determined by aggregation operators, Information Sciences 193 (2012) 153 - 162.

[37] A. Pradera, G. Beliakov, H. Bustince, B. De Baets, A review of the relationships between implication, negation and aggregation functions 
from the point of view of material implication, Information Sciences 329 (2016) 357 - 380. doi:http://dx.doi.org/10.1016/j.ins.2015.09.033.

[38] J. Pinheiro, B. Bedregal, R. H. Santiago, H. Santos, A study of (T,N)-implications and its use to construct a new class of fuzzy subsethood measure, International Journal of Approximate Reasoning 97 (2018) 1 - 16.

[39] G. P. Dimuro, B. Bedregal, Archimedean overlap functions: The ordinal sum and the cancellation, idempotency and limiting properties, Fuzzy Sets and Systems 252 (2014) $39-54$.

[40] G. P. Dimuro, B. Bedregal, H. Bustince, R. Mesiar, M. J. Asiain, On additive generators of grouping functions, in: A. Laurent, O. Strauss, B. Bouchon-Meunier, R. R. Yager (Eds.), Information Processing and Management of Uncertainty in Knowledge-Based Systems, Vol. 444 of Communications in Computer and Information Science, Springer International Publishing, 2014, pp. 252-261.

[41] J. Qiao, B. Q. Hu, On multiplicative generators of overlap and grouping functions, Fuzzy Sets and Systems 332 (2018) 1 - 24, theme: Aggregation and Operators. doi:https://doi.org/10.1016/j.fss.2016.11.010.

[42] G. P. Dimuro, B. Bedregal, On the laws of contraposition for residual implications derived from overlap functions, in: Fuzzy Systems (FUZZ-IEEE), 2015 IEEE International Conference on, IEEE, Los Alamitos, 2015, pp. 1-7. doi:10.1109/FUZZ-IEEE.2015.7337867.

[43] F. Durante, E. P. Klement, R. Mesiar, C. Sempi, Conjunctors and their residual implicators: Characterizations and construction methods, Mediterranean Journal of Mathematics 4 (3) (2007) 343-356.

[44] A. Król, Dependencies between fuzzy conjunctions and implications, in: Proceedings of EUSFLAT-LFA 2011, the 7th Conference of the European Society for Fuzzy Logic and Technology and "les rencontres francophones sur la Logique Floue et ses Applications", Atlantis Press, Amsterdam, 2011, pp. 230-237.

[45] A. Jurio, H. Bustince, M. Pagola, A. Pradera, R. Yager, Some properties of overlap and grouping functions and their application to image thresholding, Fuzzy Sets and Systems 229 (2013) $69-90$.

[46] B. Bedregal, H. Bustince, E. Palmeira, G. Dimuro, J. Fernandez, Generalized interval-valued OWA operators with interval weights derived from interval-valued overlap functions, International Journal of Approximate Reasoning 90 (2017) 1 - 16. doi:https://doi.org/10.1016/j.ijar.2017.07.001.

[47] D. Gómez, J. T. Rodríguez, J. Montero, H. Bustince, E. Barrenechea, n-dimensional overlap functions, Fuzzy Sets and Systems 287 (2016) 57 - 75, theme: Aggregation Operations.

[48] H. Bustince, E. Barrenechea, M. Pagola, F. Soria, Weak fuzzy S-subsethood measures. Overlap index, International Journal of Uncertainty, Fuzziness and Knowledge-Based Systems 14 (5) (2006) 537 - 560.

[49] G. Lucca, J. A. Sanz, G. P. Dimuro, B. Bedregal, H. Bustince, R. Mesiar, CF-integrals: A new family of pre-aggregation functions with application to fuzzy rule-based classification systems, Information Sciences 435 (2018) $94-110$.

[50] G. Lucca, J. A. Sanz, G. P. Dimuro, B. Bedregal, M. J. Asiain, M. Elkano, H. Bustince, CC-integrals: Choquet-like copula-based aggregation functions and its application in fuzzy rule-based classification systems, Knowledge-Based Systems 119 (2017) 32 - 43. doi:http://dx.doi.org/10.1016/j.knosys.2016.12.004.

[51] M. Elkano, M. Galar, J. A. Sanz, P. F. Schiavo, S. Pereira, G. P. Dimuro, E. N. Borges, H. Bustince, Consensus via penalty functions for decision making in ensembles in fuzzy rule-based classification systems, Applied Soft Computing 67 (2018) 728 - 740.

[52] M. Elkano, M. Galar, J. Sanz, A. Fernández, E. Barrenechea, F. Herrera, H. Bustince, Enhancing multi-class classification in FARC-HD fuzzy classifier: On the synergy between n-dimensional overlap functions and decomposition strategies, IEEE Transactions on Fuzzy Systems 23 (5) (2015) 1562-1580.

[53] G. Lucca, J. A. Sanz, G. P. Dimuro, B. Bedregal, H. Bustince, A proposal for tuning the $\alpha$ parameter in $\mathrm{c}_{\alpha} \mathrm{c}$-integrals for application in fuzzy rule-based classification systems, Natural Computing(in press, corrected proof online). doi:https://doi.org/10.1007/s11047-018-9678-X.

[54] H. Bustince, P. Burillo, F. Soria, Automorphism, negations and implication operators, Fuzzy Sets and Systems 134 (2) (2003) $209-229$.

[55] M. Mas, M. Monserrat, D. Ruiz-Aguilera, J. Torrens, On a generalization of the modus ponens: U-conditionality, in: J. P. Carvalho, M.-J. Lesot, U. Kaymak, S. Vieira, B. Bouchon-Meunier, R. R. Yager (Eds.), Information Processing and Management of Uncertainty in Knowledge-Based Systems, Springer International Publishing, Cham, 2016, pp. 387-398.

[56] M. Mas, D. Ruiz-Aguilera, J. Torrens, Uninorm based residual implications satisfying the Modus Ponens property with respect to a uninorm, Fuzzy Sets and Systems (2018) (In press, corrected proof). doi:https://doi.org/10.1016/j.fss.2018.09.014

[57] M. Mas, D. Ruiz-Aguilera, J. Torrens, Generalized modus ponens for (U, N)-implications, in: J. Medina, M. Ojeda-Aciego, J. L. Verdegay, D. A. Pelta, I. P. Cabrera, B. Bouchon-Meunier, R. R. Yager (Eds.), Information Processing and Management of Uncertainty in KnowledgeBased Systems. Theory and Foundations, Springer International Publishing, Cham, 2018, pp. 649-660.

[58] G. P. Dimuro, B. C. Bedregal, R. H. S. Reiser, R. H. N. Santiago, Interval additive generators of interval t-norms, in: W. Hodges, R. de Queiroz (Eds.), Logic, Language, Information and Computation, Springer Berlin Heidelberg, Berlin, Heidelberg, 2008, pp. 123-135.

[59] G. P. Farias, G. P. Dimuro, A. C. R. Costa, A fuzzy-BDI agent model for exchanges of non-economic services, based on the social exchange theory, in: Theoretical Computer Science (WEIT), 2011 Workshop-School on, IEEE, Los Alamitos, 2011, pp. 134-137.

[60] A. S. Rao, M. P. Georgeff, Modeling rational agents within a BDI-architecture, in: R. Fikes, E. Sandewall (Eds.), Proceedings og the 2nd International Conference on Principles of Knowledge Representation and Reasoning, Morgan Kaufmann, San Mateo, 1991 , pp. $473-484$.

[61] D. Adamatti, G. P. Dimuro, H. Coelho (Eds.), Interdisciplinary Applications of Agent-Based Social Simulation and Modeling, IGI Global, Hershey, 2014.

[62] F. Pereira dos Santos, D. Adamatti, H. Rodrigues, G. Dimuro, E. De Manuel Jerez, G. Dimuro, A multiagent-based tool for the simulation of social production and management of urban ecosystems: A case study on San Jerónimo vegetable garden - Seville, Spain, Journal of Artificial Societies and Social Simulation 19 (3) (2016) 12. doi:10.18564/jasss.3128.

[63] G. P. Dimuro, A. C. R. Costa, L. V. Gonçalves, A. Hübner, Centralized regulation of social exchanges between personality-based agents, in: P. Noriega, J. Vázquez-Salceda, G. Boella, O. Boissier, V. Dignum, N. Fornara, E. Matson (Eds.), Coordination, Organizations, Institutions, and Norms in Agent Systems II, Vol. 4386 of Lecture Notes in Computer Science, Springer, Berlin, 2007, pp. 338-355.

[64] G. P. Dimuro, A. R. C. Costa, L. V. Gonçalves, D. Pereira, Recognizing and learning models of social exchange strategies for the regulation of social interactions in open agent societies, Journal of the Brazilian Computer Society 17 (2011) 143-161. 